$H$ spectrum of

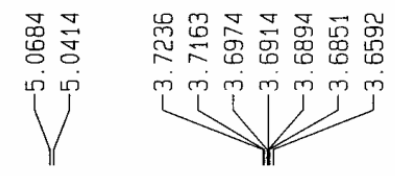<smiles>COP(=O)(OC)C(O)c1ccccc1</smiles>
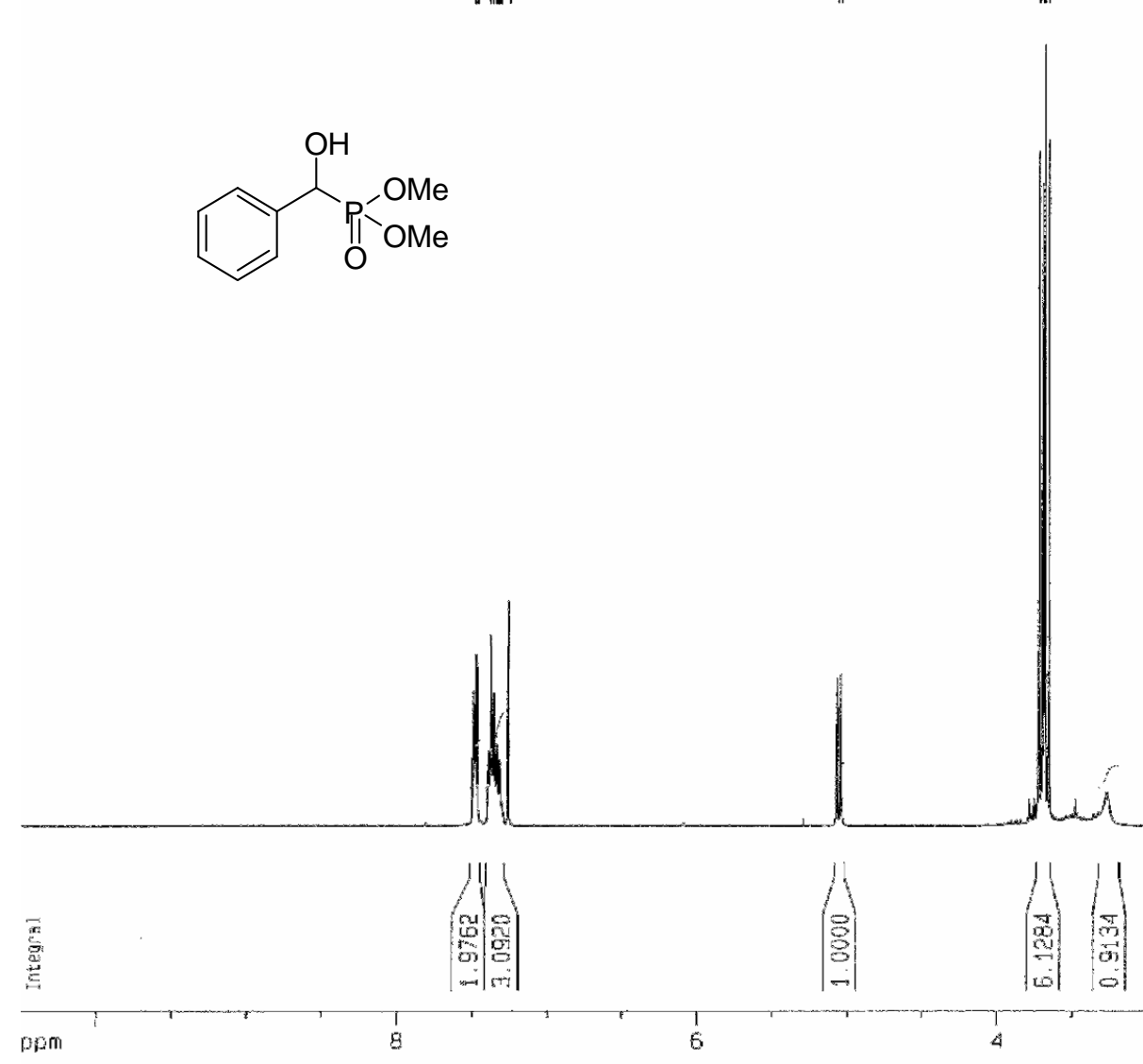

6
Current Data Parameters

NAME
EXPNO

EXPNO

F2 - Acquisition Parameter

Date_ 20051115

Time

INSTRUM $5 \mathrm{spect}$
PROBHD $5 \mathrm{~mm}$ BBO BB-1H

$\begin{array}{lr}2930 \\ \text { TD } & 16384 \\ \text { SOLVENT } & \text { CDC } 13\end{array}$

$\begin{array}{lr}\text { SOLVENT } & \text { COC13 } \\ \text { NS } & 16\end{array}$

$\begin{array}{ll}\text { DS } & 0 \\ \text { SWH } & 5995.204 \mathrm{~Hz}\end{array}$

FIDRES $\quad 0.365918 \mathrm{~Hz}$

$\begin{array}{ll}A Q & 1.3664756 \mathrm{sec}\end{array}$

JW $\quad 83.400 \mathrm{Usec}$

$\mathrm{DE}$
$\mathrm{IE}$
$\mathrm{J1}$

8.50 usec

$300.0 \mathrm{~K}$
$50000005 \mathrm{EC}$

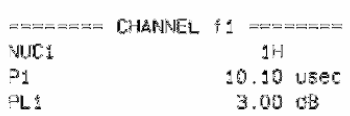

PLLO $400.1326008 \mathrm{BH}$

=2 - Proregsing parmoters

S:

SF 400.1300091 MHE

$\mathrm{H} D \mathrm{WW}$
553

$\begin{array}{cc}\angle B & 0.10 \mathrm{~Hz} \\ \mathrm{~GB} & 0 \\ =\mathrm{C} & 1.00\end{array}$

10 MMP olot paraneters

cX $\quad 20.02 \mathrm{~cm}$

$\begin{array}{ll}\text { CY } & 10.50 \mathrm{~cm}\end{array}$

$\begin{array}{ll}=1 & 4200.37 \mathrm{~Hz} \\ =2 \mathrm{P} & -0.500 \mathrm{pm}\end{array}$

$=2 \quad-200.07 \mathrm{~Hz}$

SPMCH Q $\quad 255000 \mathrm{pan} / \mathrm{cm}$ 
C13 spectrum of

워 웎 뭉

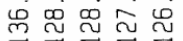

$\overbrace{1}^{\sim}$

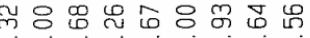

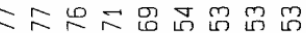

1114

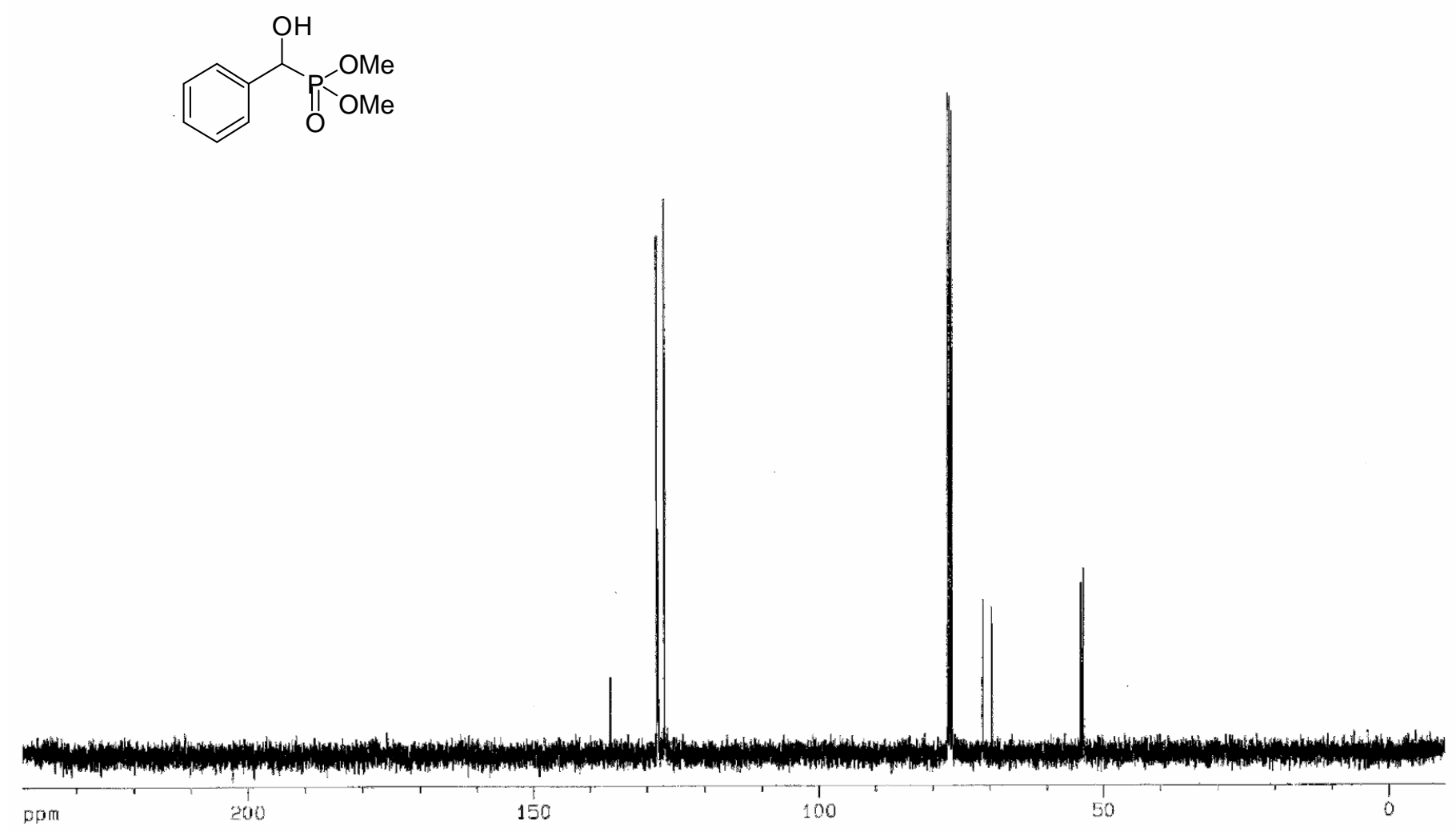

ppm

200

150

100

50

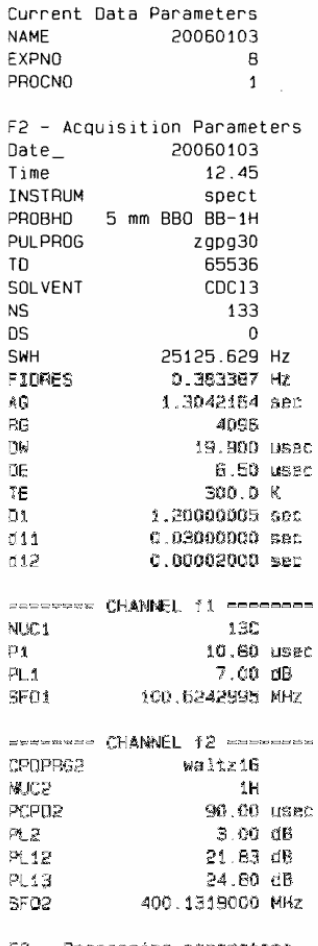

z - Pracessing paraneters

SI $\quad \begin{array}{ll}32768 \\ \text { SIF }\end{array} \quad 100.5127759 \mathrm{MHz}$

5.

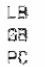

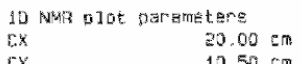

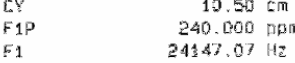

FEP $\quad 24147.07 \mathrm{~Hz}$

$\begin{array}{ll}72 & -1005.13 \mathrm{~Hz} \\ \text { PPMCM } & 12.500000 \mathrm{Dpm} / \mathrm{00}\end{array}$ 
$\mathrm{H}$ spectrum of

吾

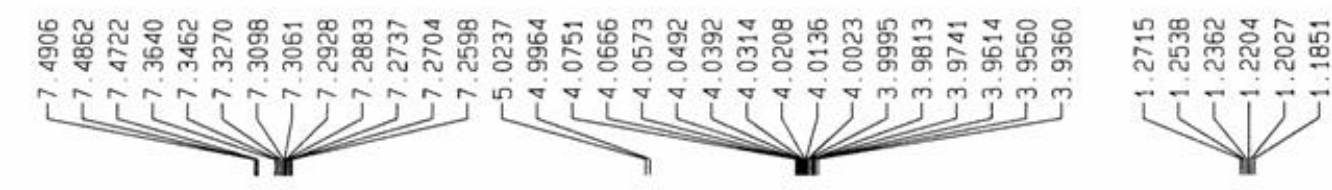

$1 \int_{0}^{\mathrm{OOEt}}$

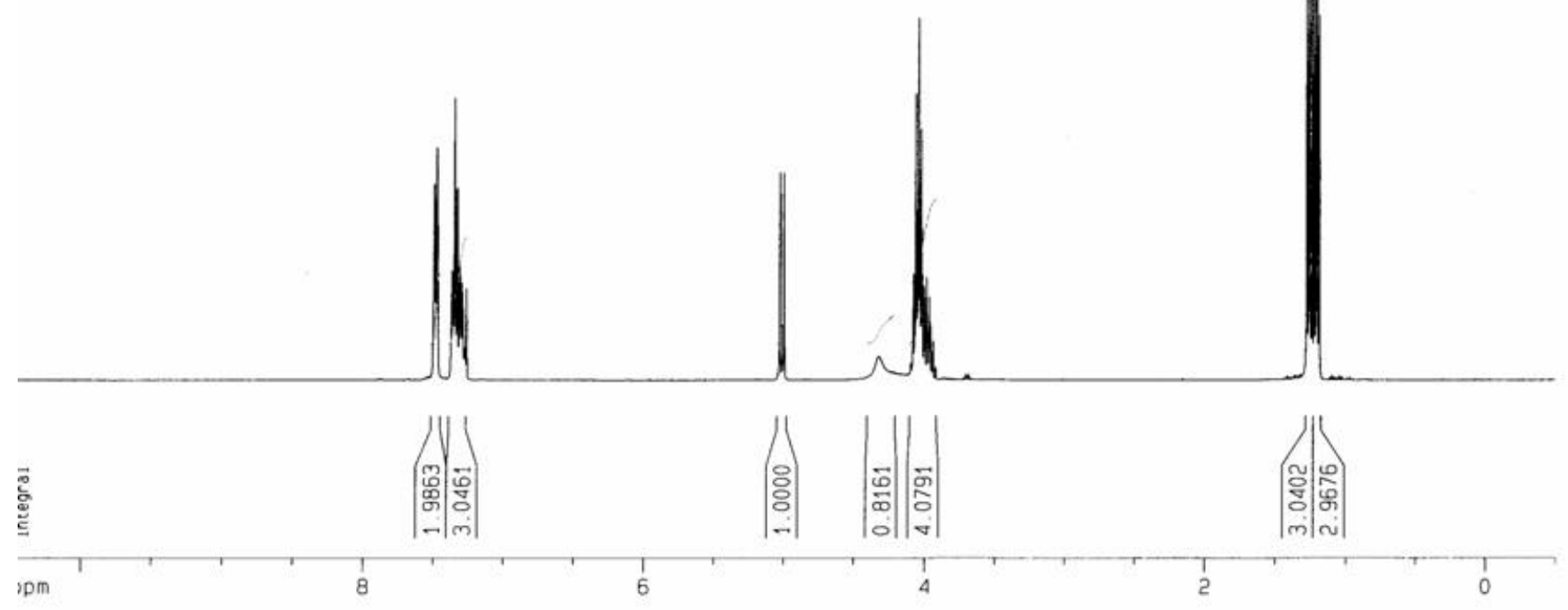

Current Data Parameter

NAME
EXPNO

PROCNO

F2 - Acquisition Parameter

Date-

Time

РАОВ 5 O $5 \mathrm{mB}$ BBO BB-1H

PULPROG 2930

$\begin{array}{ll}\text { TO } & 16384 \\ \text { SOLVENT } & \text { CDC13 }\end{array}$

NS

$\begin{array}{ll}\text { DS } & 0 \\ \text { SWH } & 5995.204 \mathrm{~Hz}\end{array}$

FQ IDES $\quad 0.365918 \mathrm{~Hz}$

AG $\quad 1.3664756$

$\begin{array}{lr}R G & 128 \\ O W & 83.400 \text { usec } \\ D E & 6.50 \text { usec }\end{array}$

TE $300.0 \mathrm{~K}$

$01 \quad 1.50000000 \mathrm{sec}$

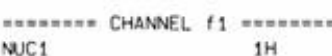

$\mathrm{P}_{1} \quad 10.10 \mathrm{usec}$

PL1 $3.00 \mathrm{~dB}$

SF01 $400.1326008 \mathrm{MHz}$

F2 - Processing parameters

SI 16384

WOW $\quad 400.1300099 \mathrm{MH}$

SSB

GB

$\mathrm{EM}$
0
$0.10 \mathrm{~Hz}$

1.00

10 NMR plot parameters

$\begin{array}{ll}\text { CX } & 20.00 \mathrm{~cm} \\ \mathrm{cy} & 10.50 \mathrm{~cm}\end{array}$

F1P $\quad 10.500 \mathrm{pDm}$

F1 $\quad 4201.37 \mathrm{~Hz}$

न2P $\quad-0.500 \mathrm{Dom}$

$\begin{array}{ll}\text { PPMCM } & 0.55000 \mathrm{Dzm} / \mathrm{cm} \\ \text { HZCM } & 220.07152 \mathrm{~Hz} / \mathrm{cm}\end{array}$ 
C13 spectrum of

윤ํㅇㅎํ



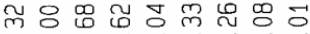

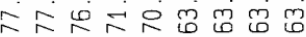

11111

m. m

ம

4

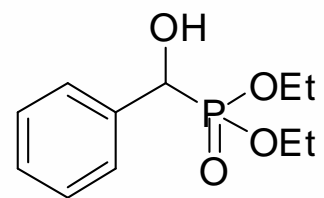

OEt

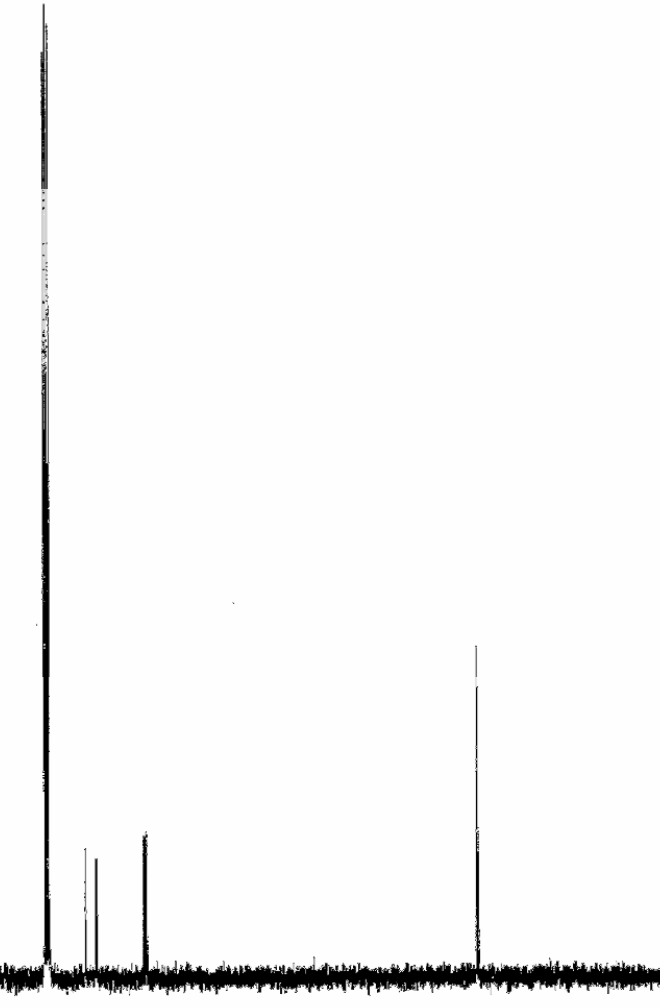

150

100

50
Current Data Parameters

EXPNO

F2 - Acquisition Parameters

Date

Time $\quad 8.24$

PROBHO $5 \mathrm{~mm}$ BBO $\mathrm{spect}$

\begin{tabular}{rr} 
PULPAOG & 29pg30 \\
TD & 65536 \\
\hline
\end{tabular}

$\begin{array}{lr}\text { SOLVENT } & \text { CDC13 } \\ \text { NS } & 501 \\ \text { DS } & 0\end{array}$

SWH $\quad 25125.629 \mathrm{~Hz}$

$0.363387 \mathrm{~Hz}$
$1.3042164 \mathrm{sec}$

$\begin{array}{ll}86 & 8000 \\ 0 \% & 19.900 \mathrm{usec}\end{array}$

TE $\quad 5.50 \mathrm{usec}$

$\begin{array}{ll}01 & 1.20300005 \mathrm{sec} \\ \text { d11 } & 0.03000000 \mathrm{sec}\end{array}$

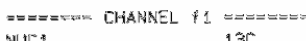

$\begin{array}{ll}\text { WUCA } & \text { 1.30 } \\ 31 & 50.80 \text { used }\end{array}$

$\begin{array}{lr}\text { PL1 } & 7.00 \mathrm{~dB} \\ \text { Se01 } & 100.624 .2995 \mathrm{MHZ}\end{array}$

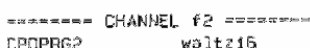

$\begin{array}{ll}\text { NUCE } & 1 \mathrm{H} \\ \text { PCFD? } & 90.00 \mathrm{usec}\end{array}$

PL.?

PL13
SFO2

F2 - Processing pinnameters

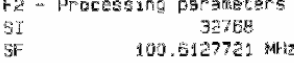

때에

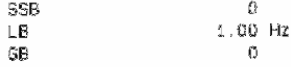

10 filn plot parameters

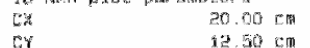

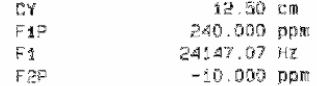

$-10.005 \mathrm{ppm}$
$\mathrm{Fe}$

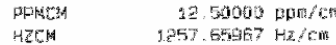


$H$ spectrum of

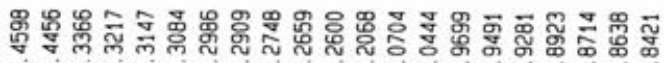

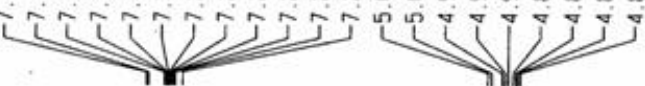
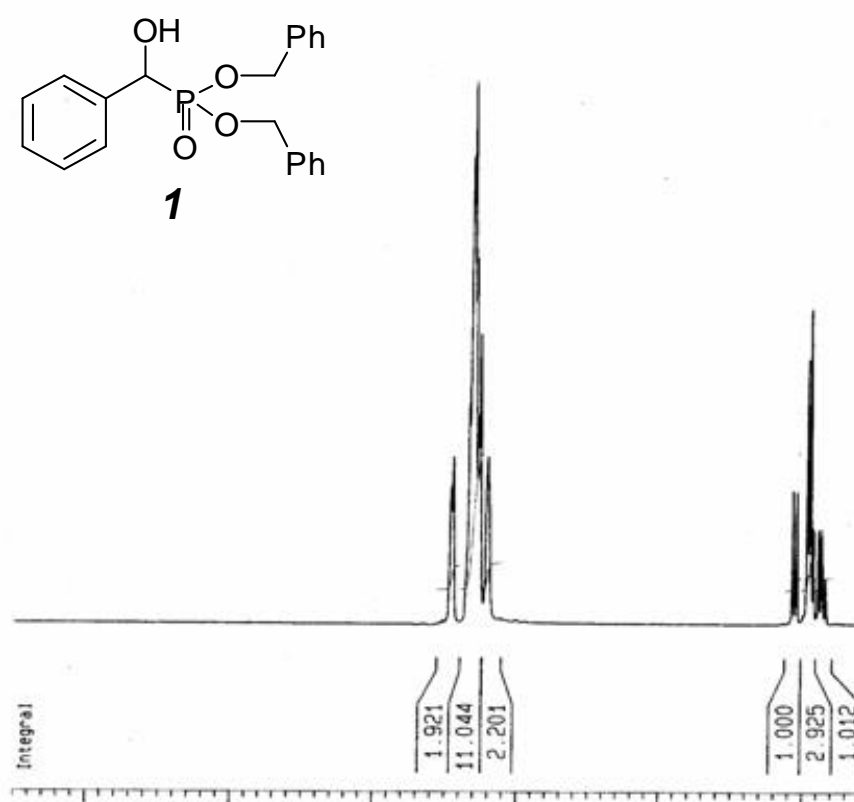

Current Data Parameters
NAME
EXPNO
PROCNO

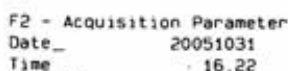

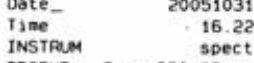

PAOBHO $5 \mathrm{~mm}$ BBO BB-1
PULPROG

$\begin{array}{lr}2930 \\ \text { TO } \\ \text { SOUVNT } & 16384 \\ \text { NS } & \text { COC13 }\end{array}$

$\begin{array}{lc}\text { NS } & 16 \\ \text { OS } & 0 \\ \text { SHH } & 5995.204 \mathrm{~Hz}\end{array}$

$\begin{array}{ll}\text { Fiofes } & 0.365918 \mathrm{~Hz} \\ { }_{\text {AO }} & 13664756 \mathrm{sec}\end{array}$

$\begin{array}{lr}\text { AG } & 1.3664756 \mathrm{sec} \\ \text { OW } & 322.5 \\ \text { OW } & 83.400 \mathrm{usec}\end{array}$

$\begin{array}{ll}\text { TE } & 3000 \mathrm{~K} \\ \mathrm{D} 1 & \mathrm{~K}\end{array}$

NUC: 1 .... CHANNEL, +1, .........

NUC1 $\quad 10.10$ usec

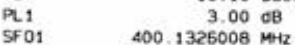

F2 - Processing parameters

$\begin{array}{ll}\text { SI } & 16384 \\ \text { SF } & 400,1300099\end{array}$

$\begin{array}{lc}\text { NOW } & \text { EM } \\ \text { SSB } & 0 \\ \text { LB } & 0.10 \mathrm{~Hz}\end{array}$

PC $\quad 1.00$

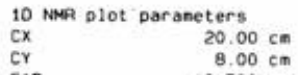

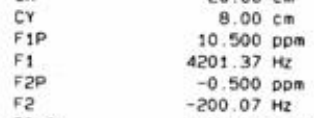

$0.55000 \mathrm{ppm} / \mathrm{cm}$
$220.07152 \mathrm{~Hz} / \mathrm{cm}$ 
C13 spectrum of

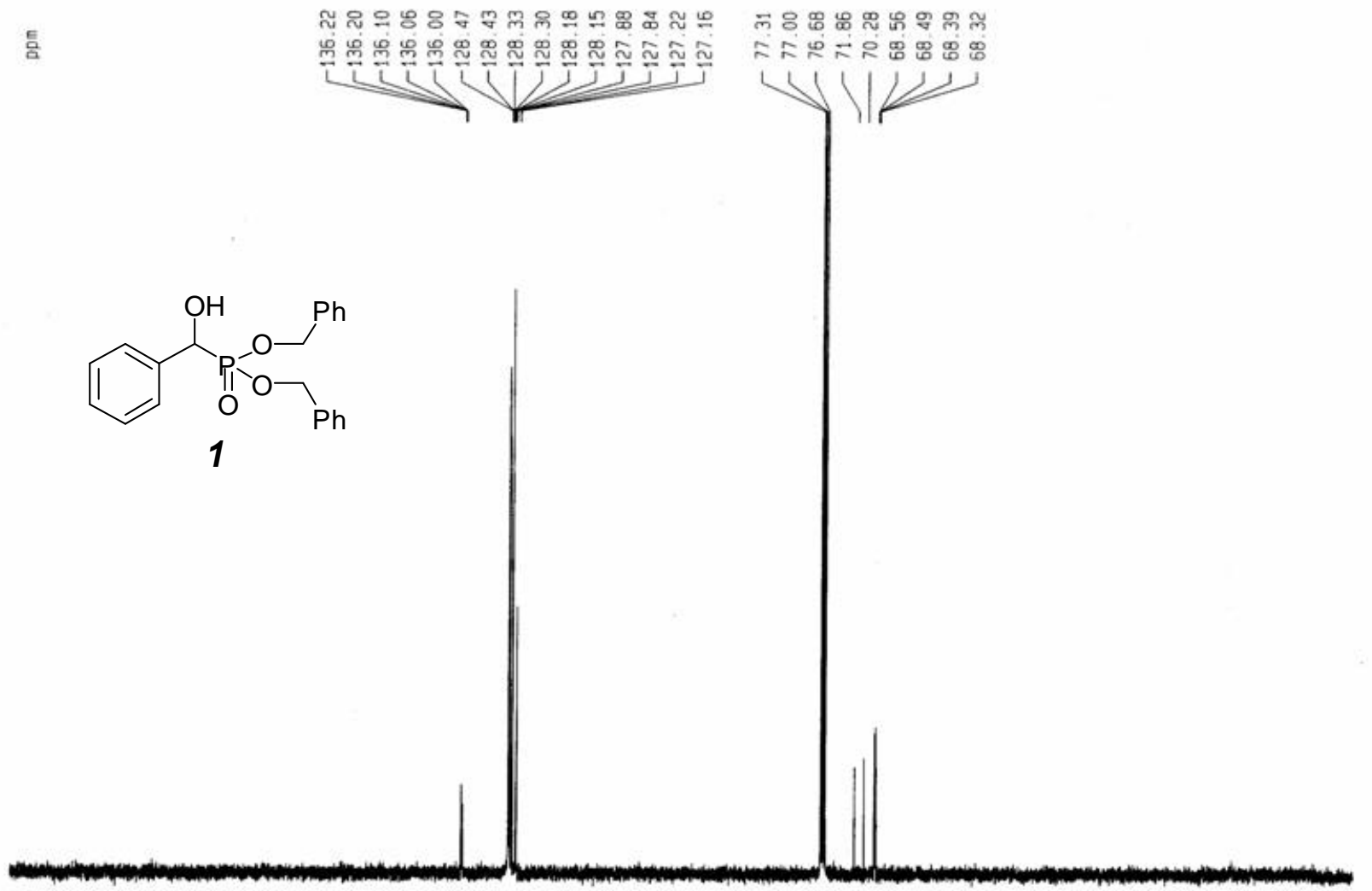

Nure
EXPN
PAOCiO

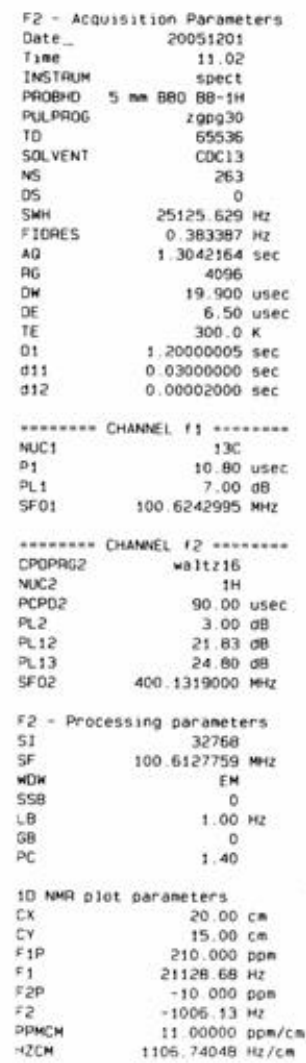


$H$ spectrum of

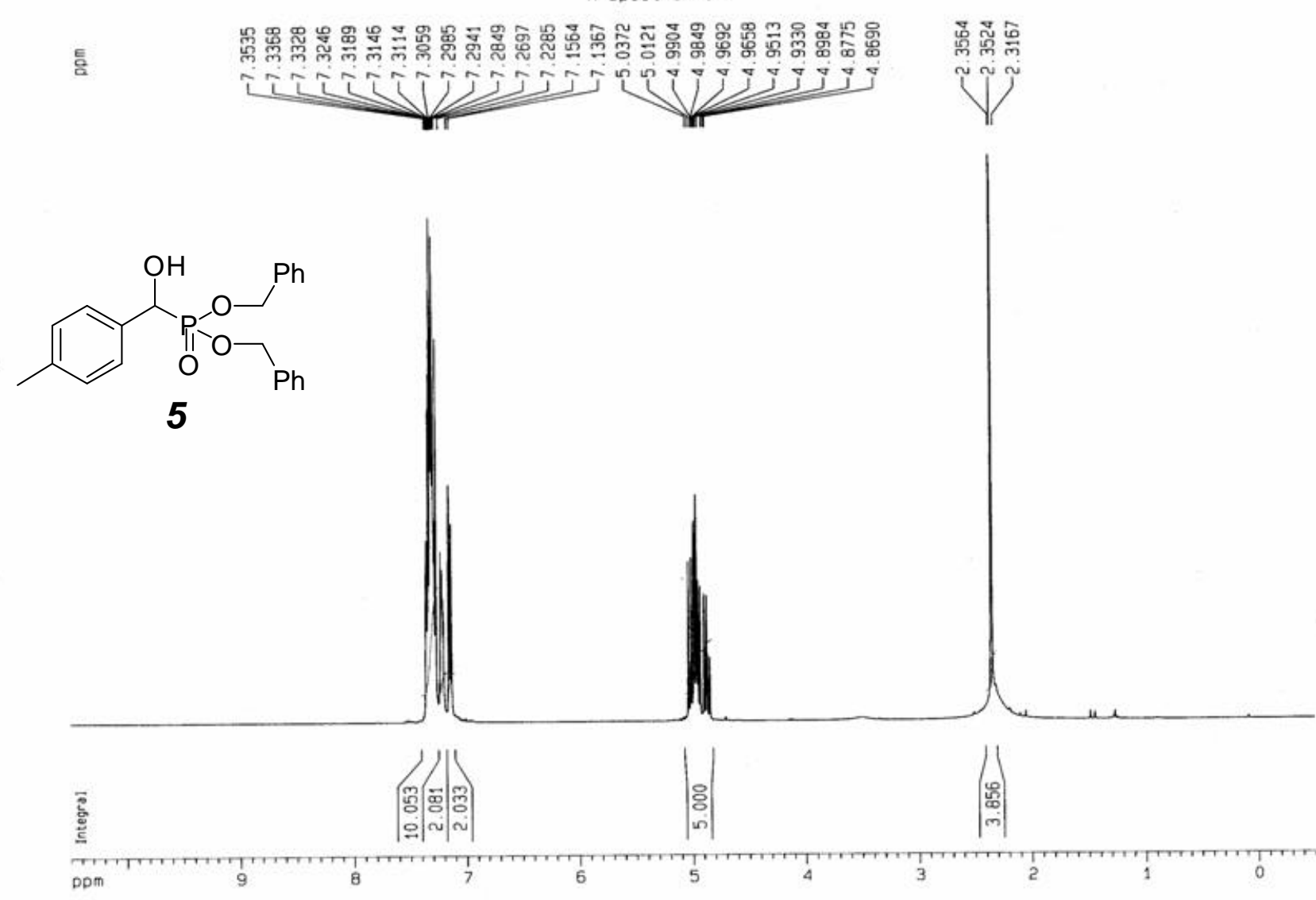

Current data Parameters
NAME

EXPNO

2 . Acquisition Parameters

19.15
INSTRUM

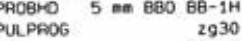

ID

$\begin{array}{lr}\text { SOLVENT } & \text { COCC13 } \\ \text { NS } & 32 \\ \text { OS } & 0\end{array}$

$\begin{array}{ll}\text { SWH } & 5995.204 \mathrm{~Hz} \\ \text { EIDPES } & 0.365918 \mathrm{~Hz}\end{array}$

$\begin{array}{ll}A 0 & 1.3654756 \mathrm{sec} \\ { }^{A 6} & 256\end{array}$

$\begin{array}{rr}\text { DW } & \begin{array}{r}256 \\ \text { DE }\end{array} \\ \text { DE } & 6.50 \text { usec } \\ \text { TEsec }\end{array}$

$\begin{array}{ll}\pi & 300.0 \mathrm{~K} \\ 01 & 1.50000000 \mathrm{sec}\end{array}$

$\begin{array}{lll}\text { NWCA CHANEL H1 } & 1 \mathrm{H} \\ \mathrm{P}_{1} & 10.10 \mathrm{usec}\end{array}$

$\begin{array}{ll}P l_{1} & 3.0008 \\ S F 01 & 400.1326008\end{array}$

52 - Processing parameters

$\begin{array}{ll}\text { SI } & 16384 \\ \text { SF } & 400.1300050 \\ \text { HOW } & \text { EMt }\end{array}$

$\underset{C B}{S B} \quad 0.10 \mathrm{~Hz}$

10 NMR plot paraneters

$\begin{array}{ll}C X & 20.00 \mathrm{~cm} \\ C Y & 10.50 \mathrm{~cm}\end{array}$

$\begin{array}{rr}\text { Fip } & 10.500 \mathrm{~cm} \\ F_{1} & 4201.37 \mathrm{~Hz}\end{array}$

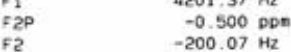

PPMCM
HZCM
$220.075000 \mathrm{DDD} / \mathrm{cm}$ 
C13 spectrum of

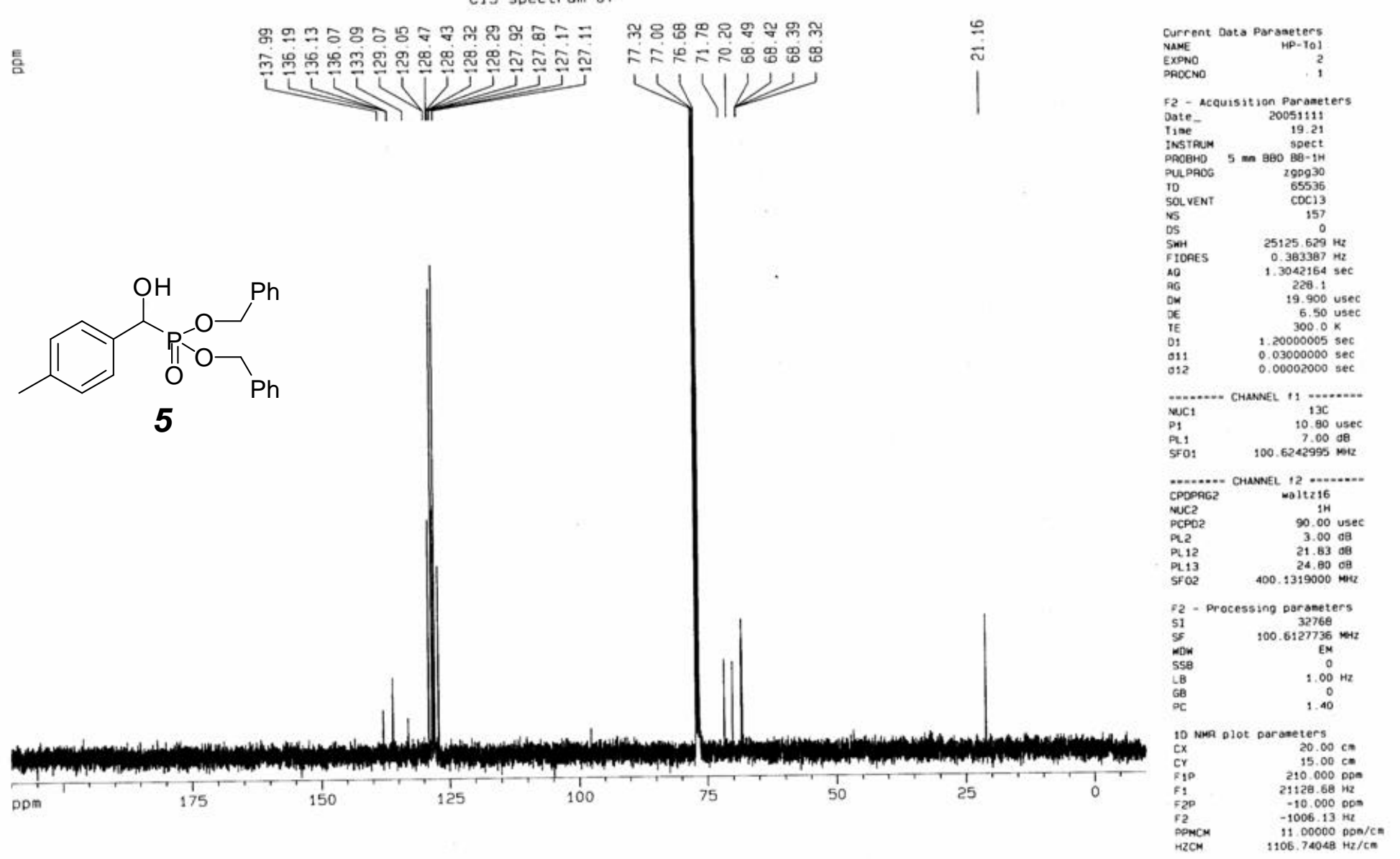


H spectrue of

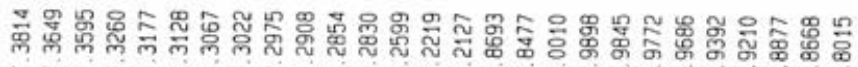

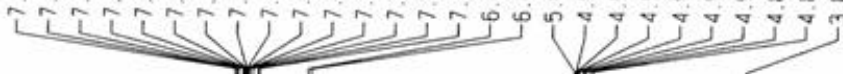
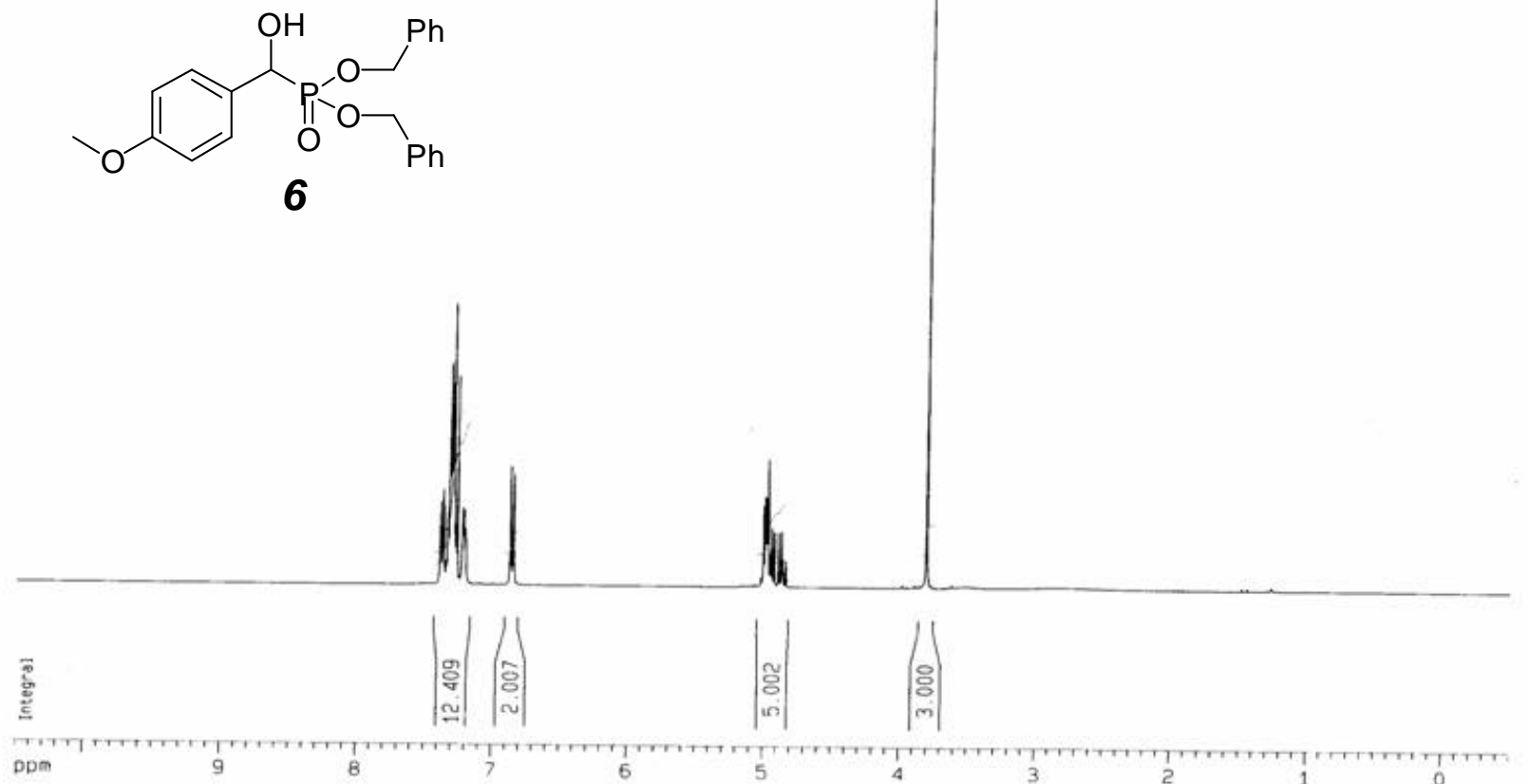

pDo
Current Dato Parameters
NaNe
EXPQO

EXPNO
SROCNO

F2 - Acquisition parameters

Date.

TNSTRer 19.38 PRDBHO $5 \mathrm{~mm} 880 \mathrm{sec} 8 \mathrm{~B}-1 \mathrm{H}$

to

NS $\quad 64$

SWH $\quad 5995.204 \mathrm{He}$

AO $\quad 0.365918 \mathrm{~Hz}$

\begin{tabular}{rr}
$A Q$ & $1.3664756 \mathrm{sec}$ \\
$\mathrm{AG}$ & 32.5 \\
$\mathrm{OW}$ & 83.400 use \\
\hline
\end{tabular}

$\begin{array}{lr}\text { DE } & 5.50 \text { use } \\ \text { IE } & 300.0 \mathrm{~K} \\ 01 & 1.50000000 \mathrm{sec}\end{array}$

.......... CMANwEL 11 .........

$\begin{array}{ll}\text { NuC1 } & 1 \mathrm{H} \\ \text { P1 } & 3.00 \mathrm{usec} \\ \text { P. } & 3.00 \mathrm{~dB}\end{array}$

$\begin{array}{lr}\text { F2 } & \text { - Processing Darameters } \\ \text { S1 } & 16384 \\ \text { SF } & 400.1300099 \mathrm{NHZ}\end{array}$

NOW $\quad 400.1300099 \mathrm{MHz}$

$\llcorner\mathrm{B}$

PC $\quad 1.00$

10 NaA olot paraneters
Cx

$\begin{array}{lr}\mathrm{Cr} & 10.50 \mathrm{~cm} \\ F_{1} & 10.500 \mathrm{ppm} \\ F_{1} & 4201.37 \mathrm{~Hz}\end{array}$

-0.500
$-2000 \mathrm{~m}$

$\begin{array}{rl}-200.07 \mathrm{~Hz} & \mathrm{~Hz} \\ 0.55000 & \mathrm{ppm} / \mathrm{cm}\end{array}$ 
C13 spectrum of
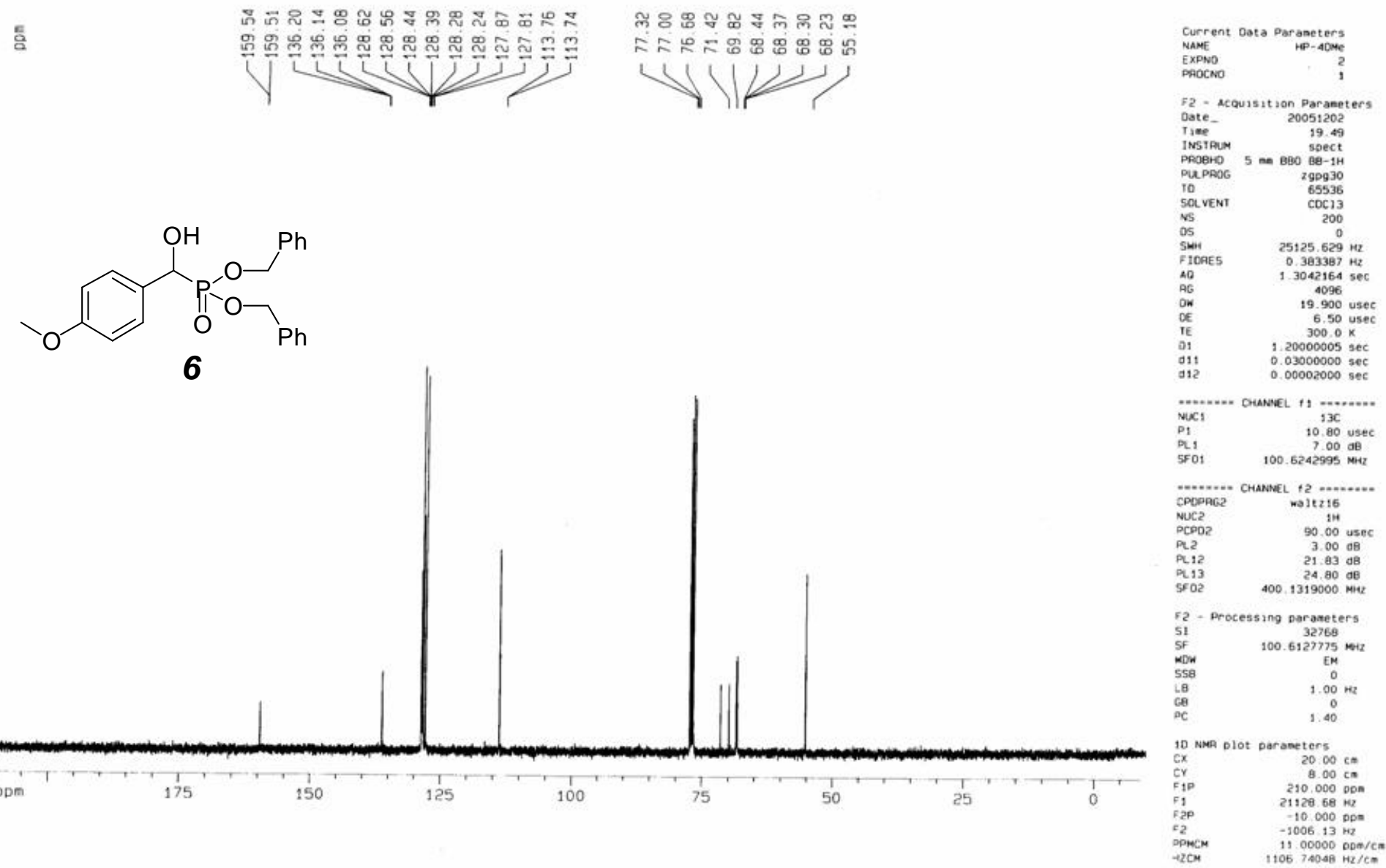
$\mathrm{H}$ spectrum of

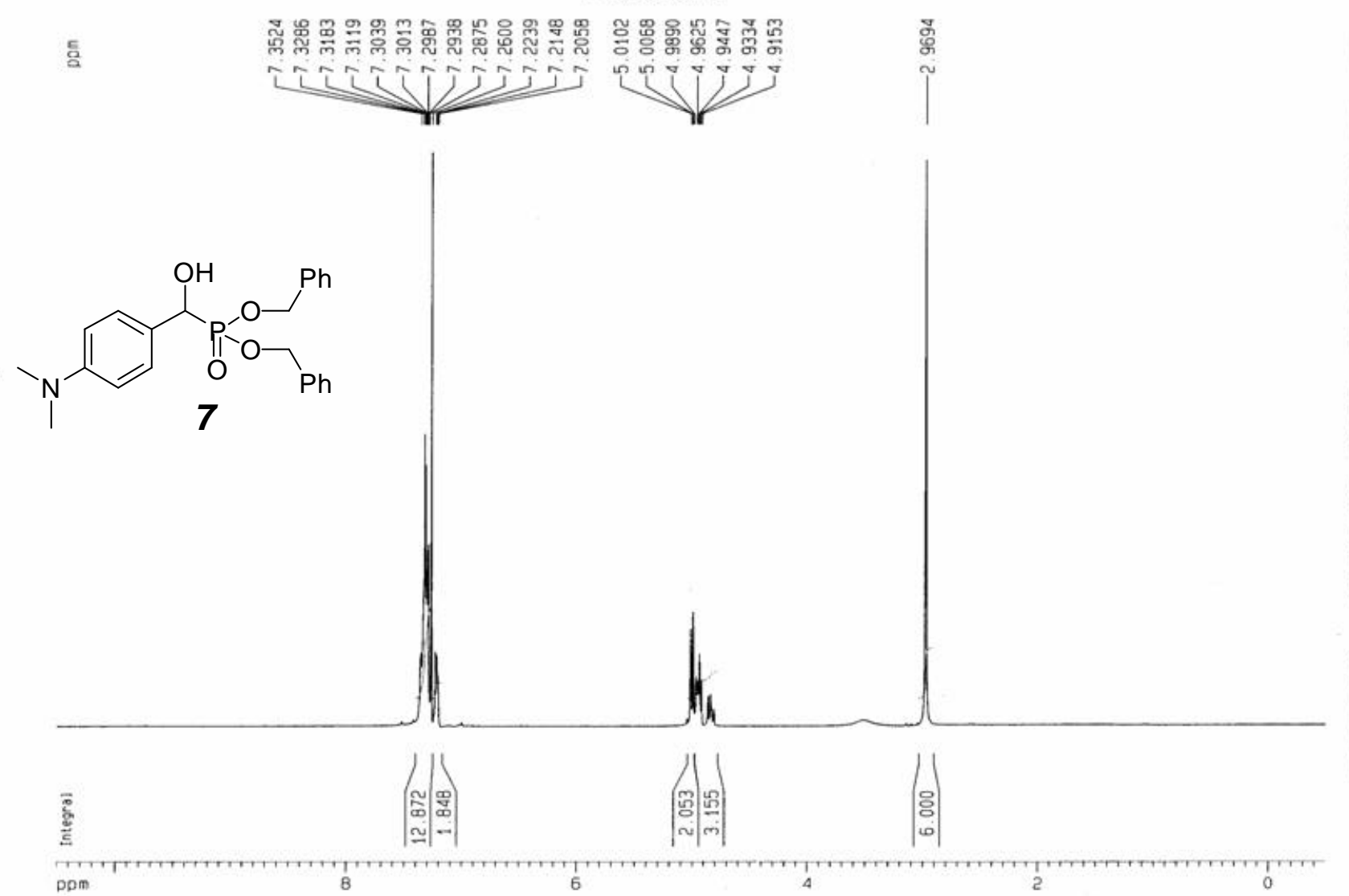

Current Data Parameters

EXPNO

F2- Acquisition Paraneters
Date- 20051123

Time

PPOBHO 5 seect

PULPAOG
TD

N5 54

$\begin{array}{ll}\text { SWH } & 5995.204 \mathrm{~Hz} \\ \text { EDRES } & 0.365919 \mathrm{~Hz}\end{array}$

$\begin{array}{ll}A 0 & 1.3664756 \mathrm{sec} \\ \text { AG } & 362\end{array}$

$\begin{array}{lr}\text { AG } & 362 \\ \text { OW } & 83.400 \mathrm{usec} \\ \text { OE } & 5.50 \mathrm{usec}\end{array}$

$\begin{array}{ll}\text { IE } & \quad 300.0 \mathrm{~K} \\ 01 & 1.50000000 \mathrm{sec}\end{array}$

NuC1 CHAnel. 11 ..........

$\begin{array}{lr}\text { P1 } & 10.10 \text { use } \\ \text { PL1 } & 3.00 \mathrm{~dB} \\ \text { SFO1 } & 400.1326008 \mathrm{Mar2}\end{array}$

F2 - Processing parameters
S1
SF 400.16304
SF

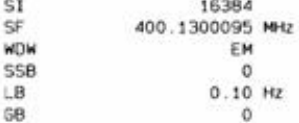

10 Nar plot parameters
$\mathrm{Cx}$
$\mathrm{CY}$
$\mathrm{CY}$

$\begin{array}{lr}10.50 \mathrm{~cm} \\ \mathrm{~F} 1 \mathrm{PP} & 10.500 \mathrm{DD} \\ \mathrm{E} & 4201.37 \mathrm{~Hz}\end{array}$

$-0.500 \mathrm{DD}$

$\begin{array}{rl}0.55000 & \mathrm{ppm} / \mathrm{cm} \\ 220.07150 \mathrm{~Hz} / \mathrm{cm} & -5\end{array}$ 
C13 spectrum of

Current Data Parabeters
NAME
HP.NACD-res

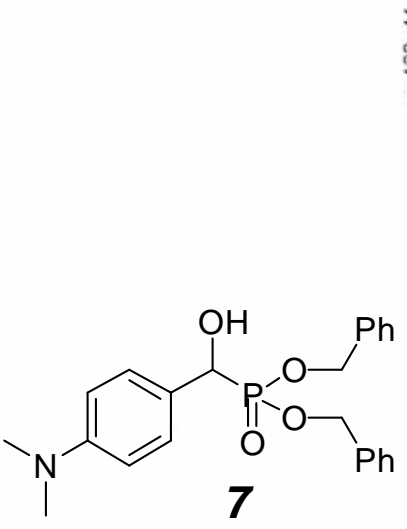

VIKK

ExpNo

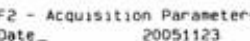

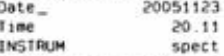

ppoero
Pueproc

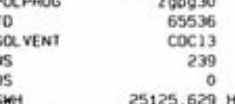

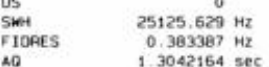

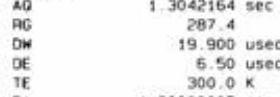

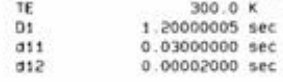

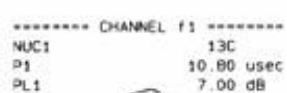

91.
sfor

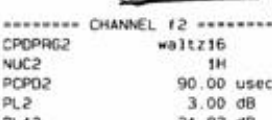

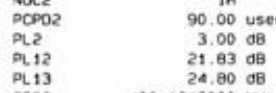

(139000

22 - Processing paraseteres

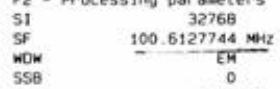

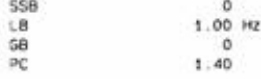

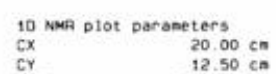
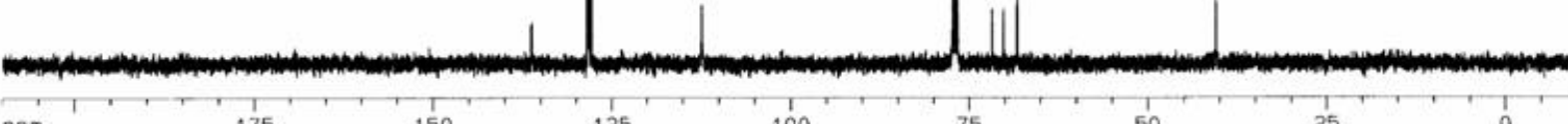

150

125

100

50

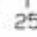

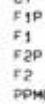

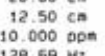

$-10.000 \mathrm{pon}$
$-1006.13 \mathrm{me}$
-130006

11.00000
$1106.70060 \mathrm{pg} / \mathrm{cm} / \mathrm{cm}$ 


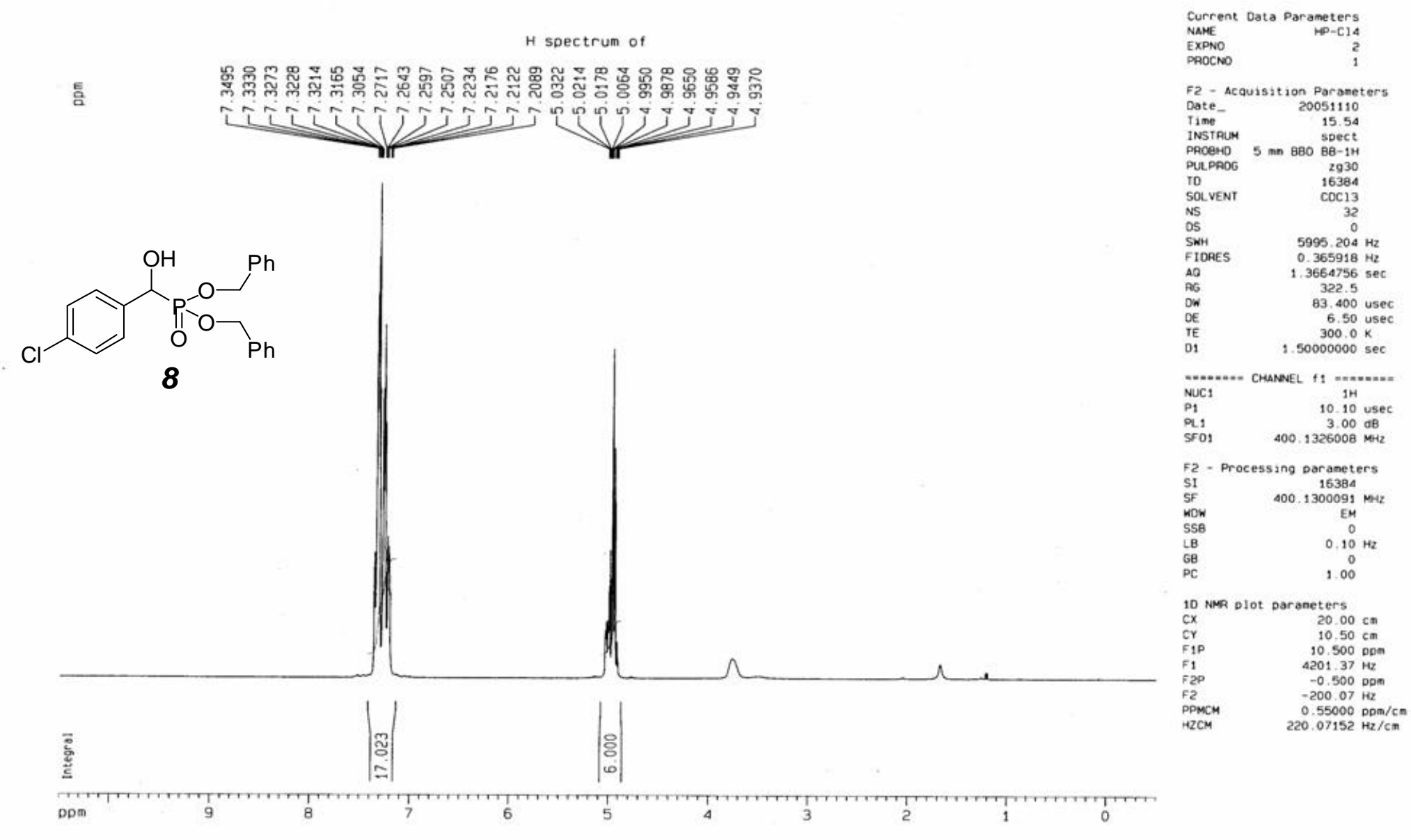


C13 spectrum of
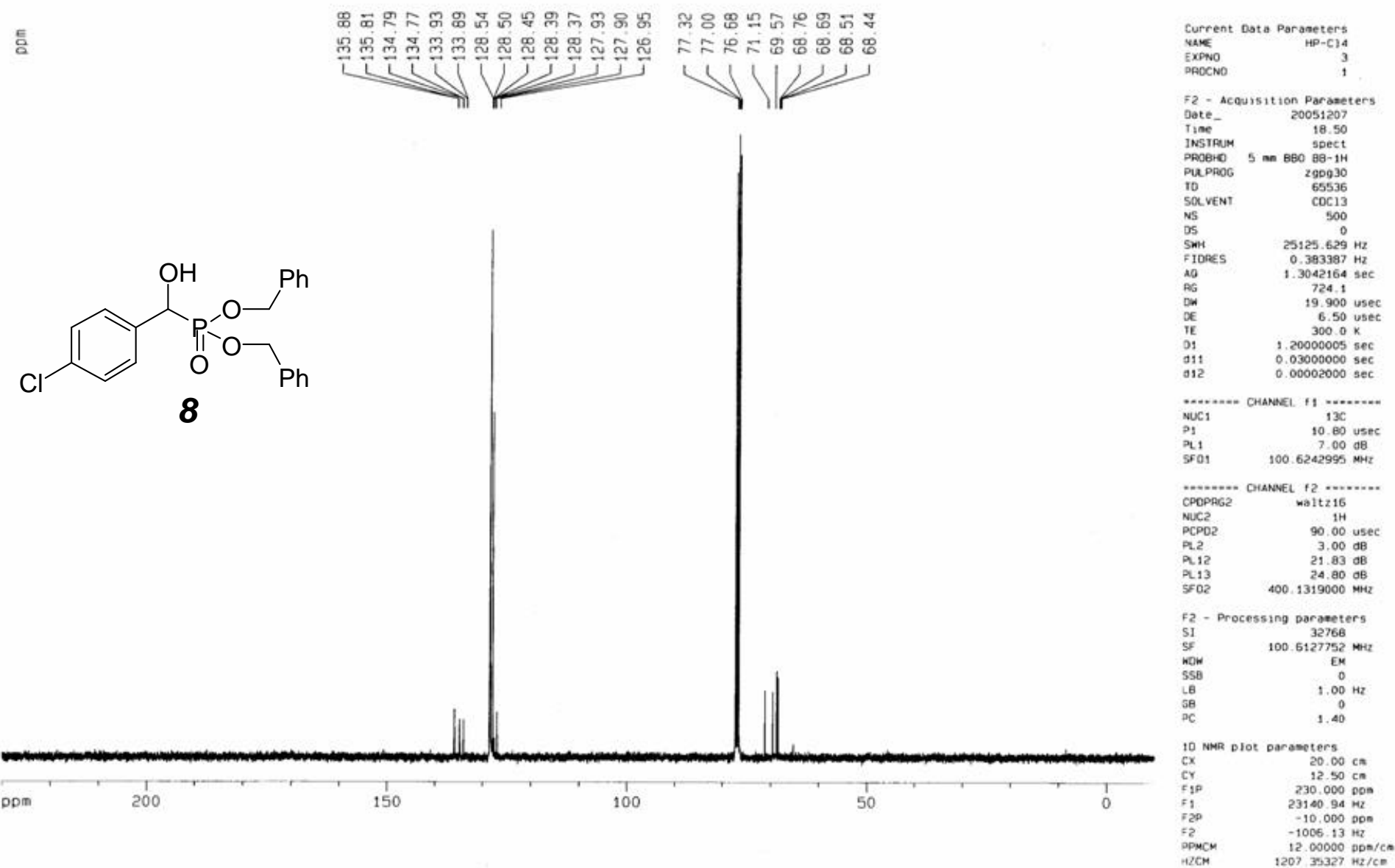


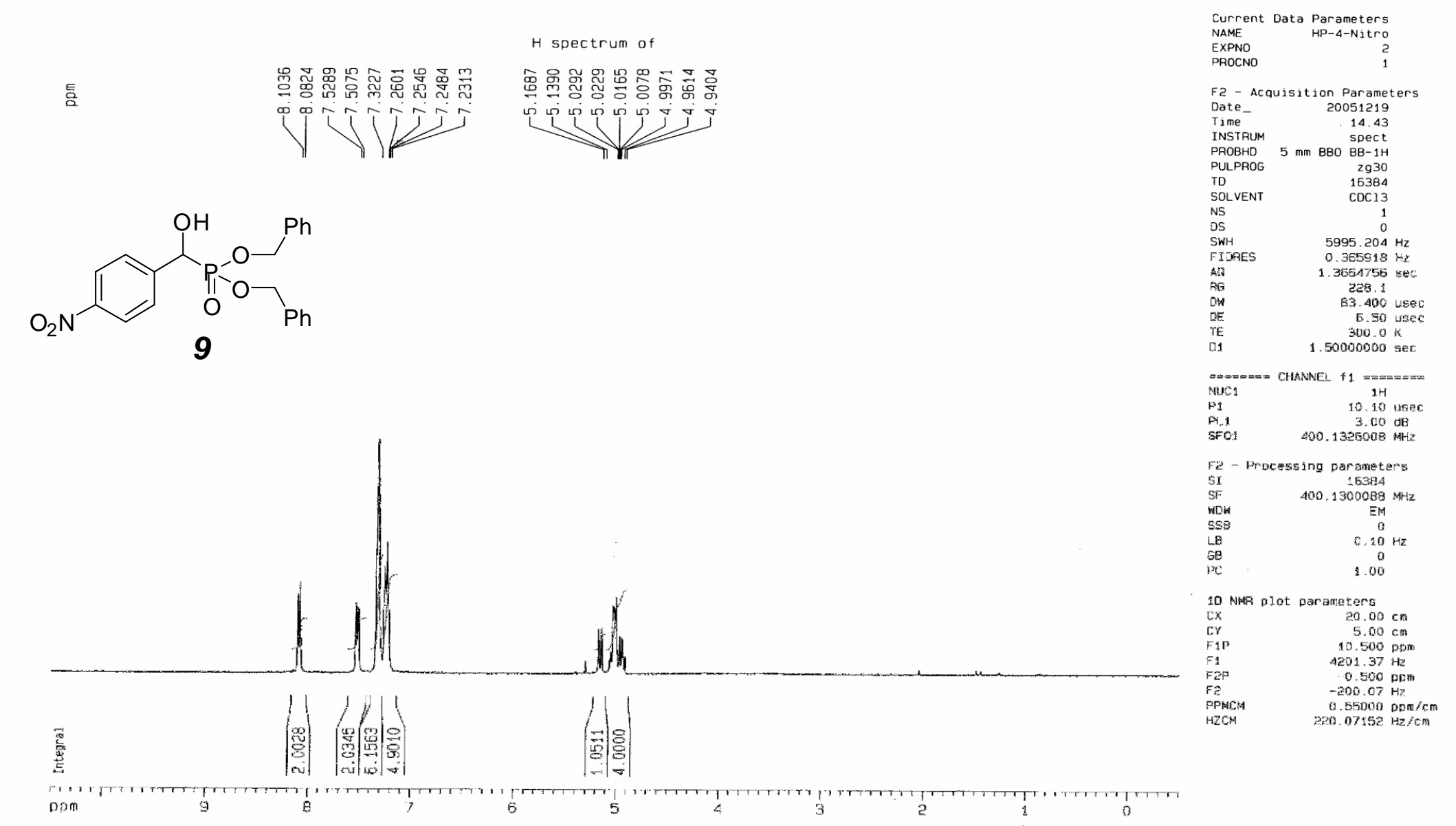


13 spectrum of

豆<smiles>O=C(OCc1ccccc1)C(O)c1ccc([N+](=O)[O-])cc1</smiles>

9

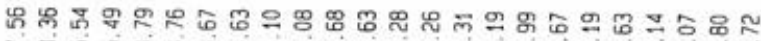

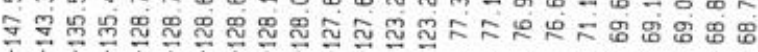

Curre
Nave
ExpeNo

EXPNO
PAOCin

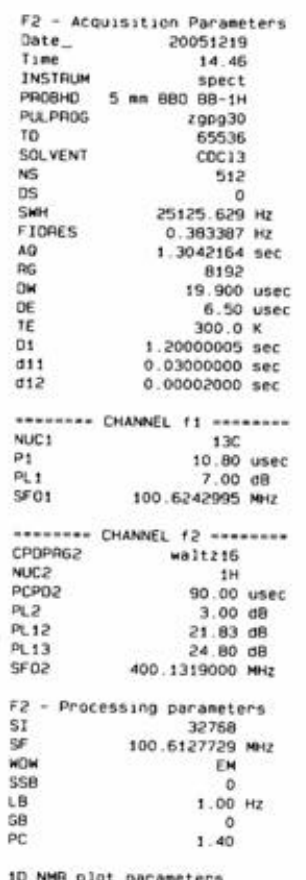

10 Nur plot parameters
$20.00 \mathrm{ch}$
20

50

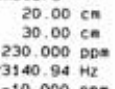

200

150
100

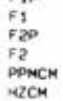

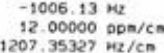




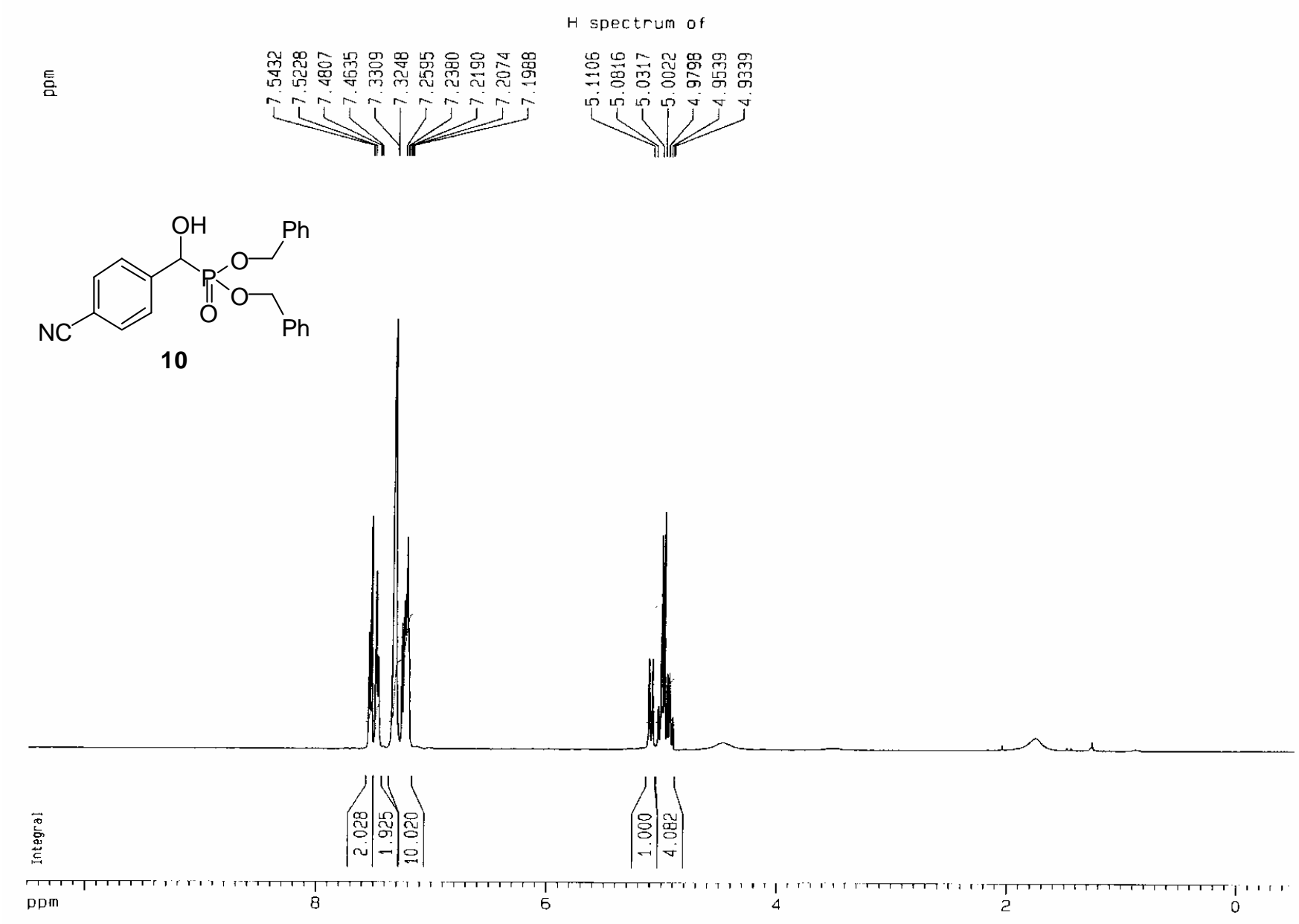

Current Data Parameters

NAME HP-4-Cyano

F2 - Acquisition Parameter

Date_ 20060315

$\begin{array}{lr}\text { TIme } & 17.12 \\ \text { INSTRUM } & \text { spect } \\ \text { PROBHD } & 5 \mathrm{~mm} B \mathrm{BQ}-1 \mathrm{H}\end{array}$

PULPROG

PULPRO
TO

$\begin{array}{ll}\text { SOLVENT } & 16384 \\ \text { NS } & \text { COC13 }\end{array}$

0
DS $\quad 5995.204 \mathrm{~Hz}$

$\begin{array}{ll}\text { SHH } & 5995.204 \mathrm{~Hz} \\ \text { FIDRES } & 0.365918 \mathrm{~Hz}\end{array}$

$\begin{array}{lc}A Q & 1.3654756 \mathrm{sec} \\ \mathrm{RG} & 287.4\end{array}$

$\begin{array}{lr}\mathrm{DW} & 83.400 \mathrm{usec} \\ \mathrm{DE} & 6.50 \mathrm{usec}\end{array}$

11 $\quad 1.50000000 \mathrm{~K}$

$==s====$ CHANNEL $f,==== \pm= \pm$

$\begin{array}{lll}\text { NUC1 } & 1 \mathrm{H} \\ \mathrm{P} 1 & 10.10 \mathrm{usec}\end{array}$

$\begin{array}{lr}\text { P1 } & 10.10 \text { usec } \\ \text { PL1 } & 3.00 \mathrm{~dB} \\ \text { SF01 } & 400.1326008 \mathrm{MHz}\end{array}$

F2 - Processing parameters

$\begin{array}{lr}\text { SI } & 16384 \\ \text { SF } & 400.1300099 \mathrm{MHz}\end{array}$

WOW
SSB

10 NMP plot parameters

$\begin{array}{lr}\text { CX } & 20.00 \mathrm{~cm} \\ \text { CY } & 8.00 \mathrm{~cm} \\ \text { F1P } & 10.500 \mathrm{pmm}\end{array}$

$\begin{array}{ll}\text { F1 } & 10.500 \mathrm{ppm} \\ F 2 \mathrm{P} & 4201.37 \mathrm{~Hz}\end{array}$

$\begin{array}{ll}-0.500 \mathrm{ppm} \\ \mathrm{FP} & -200.06 \mathrm{~Hz}\end{array}$

PPMCM
HZCM
$220.075000 \mathrm{Dpm} / \mathrm{cm}$ 
C13 spectrum of

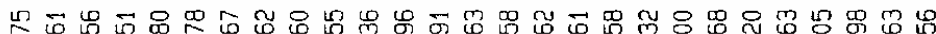

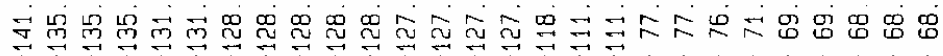
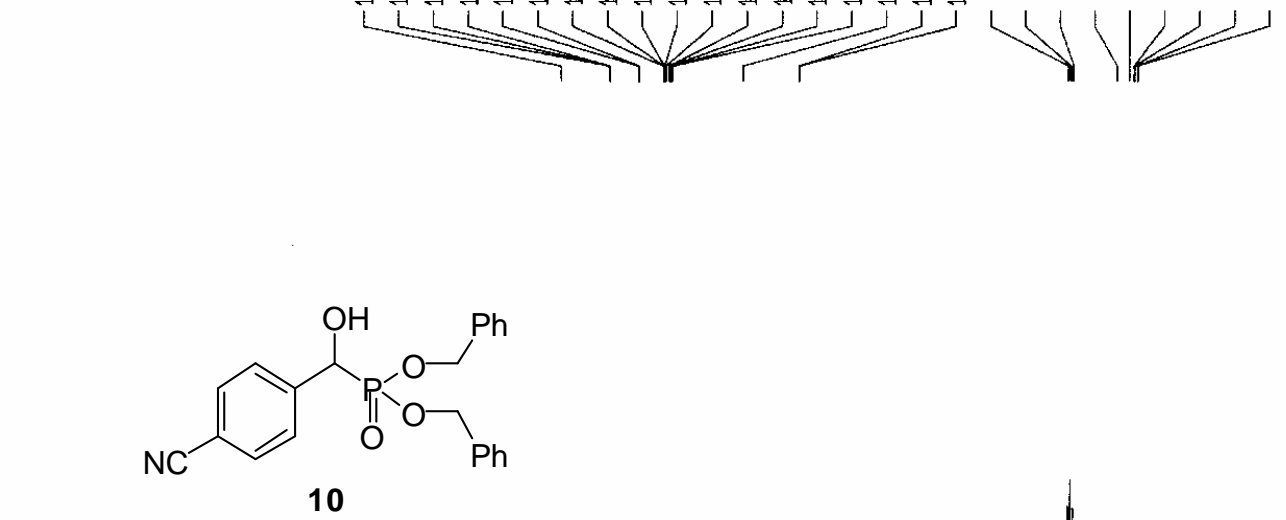

10
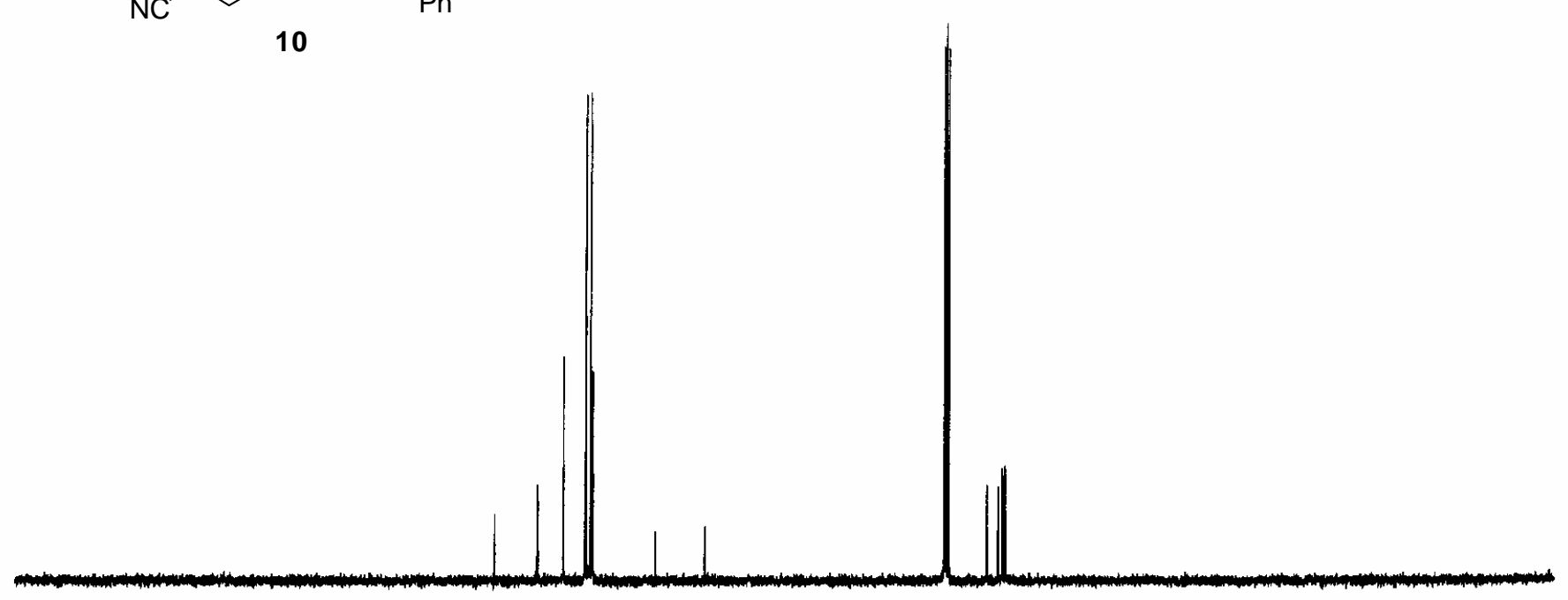

ppm

175

150

125

100

75

50

25

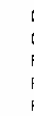

Current Dat a Parameters

EXPNO

F2 - Acquisition Paraneters

Parameters

Date

INIme

PAOBH

TD POLOE

SOLVEN
NS

NS
DS
SWH

DS
SWH
F IDRES
AQ

AQ

AQ
RG
$\mathrm{OH}$
$\mathrm{DE}$
$\mathrm{DE}$

ON
DE
TE
$D 1$

01
d1
a

d11

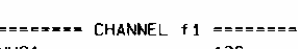

NuC1 130

$\begin{array}{lr}\text { PL1 } & 10.80 \text { usec } \\ \text { PL1 } & 7.00 \text { dB }\end{array}$

$===== \pm$ CHANNEL $+2=====-=$
CPDPAG2

NuC2 13

$\begin{array}{ll}\text { PCPD2 } & 90.00 \text { used } \\ \text { PL2 } & 3.00 \mathrm{~dB} \\ \text { PL12 } & 21.83 \mathrm{~dB}\end{array}$

PL13
PLO2

2 - Processing parameters

F2 - Processing parameters
SI
SI
SF

$\begin{array}{lc}\text { SF } & 100.6127775 \mathrm{MHZ} \\ \text { WOW } & \mathrm{EM} \\ \text { SSB } & 0\end{array}$

$\begin{array}{lc}\mathrm{SSB} & 0 \\ \mathrm{LB} & 1.00 \mathrm{~Hz} \\ 6 \mathrm{~B} & 0 \\ \mathrm{O} & 1.40\end{array}$

1D NMP plot parameters

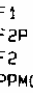

$20.00 \mathrm{~cm}$
$8.00 \mathrm{~cm}$

$210.000 \mathrm{ppm}$
$21128.68 \mathrm{~Hz}$

$-10.000 \mathrm{ppm}$

$-1006.13 \mathrm{~Hz}$
$11.00000 \mathrm{Dgm} / \mathrm{c}$

$\mathrm{HZCM}$ 
$H$ spectrum of

言

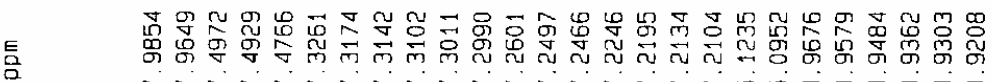
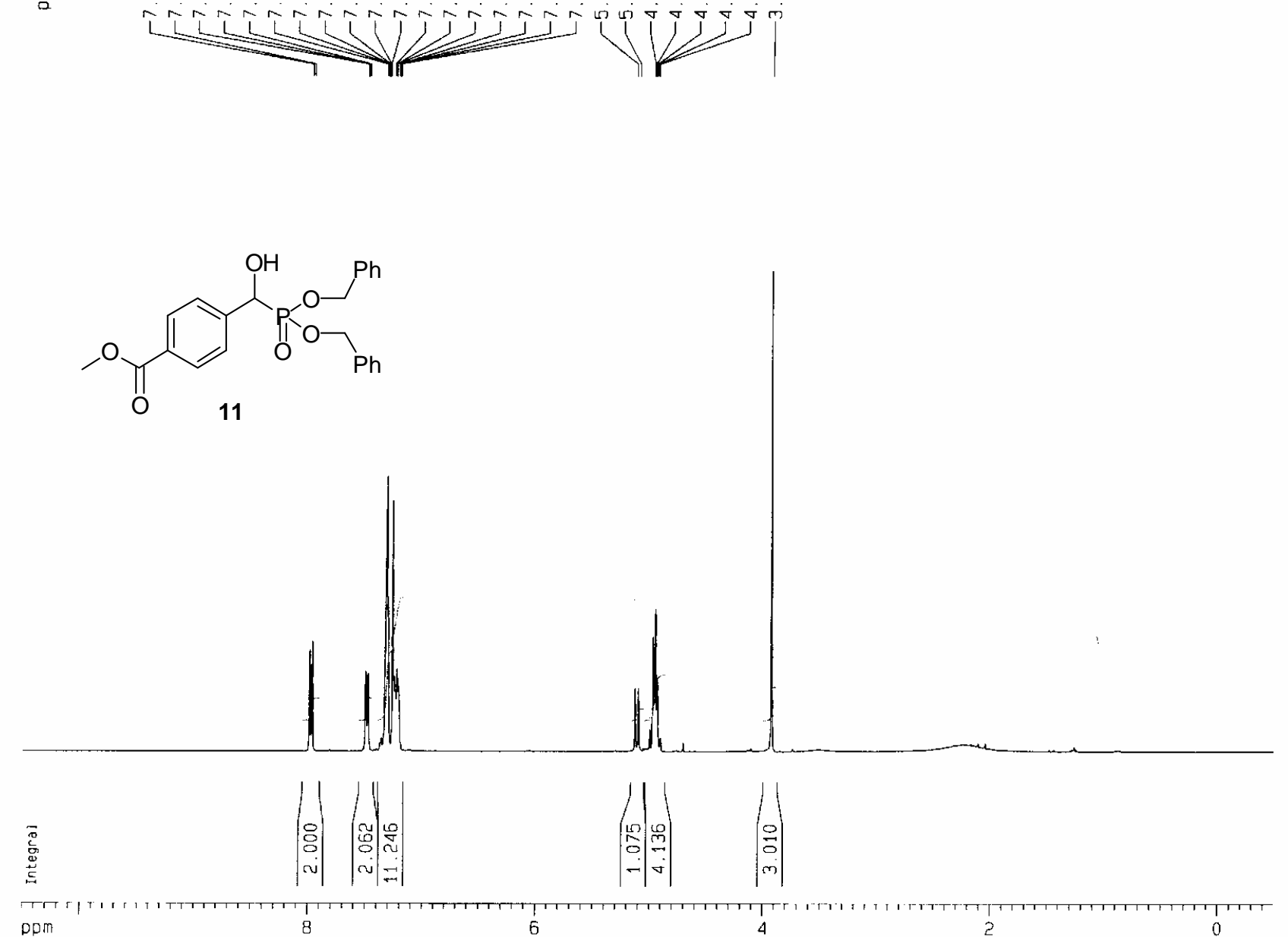

Current Data Parameters

NAME HP-AMethylbenz

PROCNO

F2 - Acquisition Parameters

Date

Time

PROBHD $5 \mathrm{~mm}$ BBO BB- $\mathrm{HH}$

PULPROG

SOLVENT

$\begin{array}{lrl}\text { NS } & \text { EO } \\ \text { SS } & \text { EOLNT }\end{array}$

SWH $\quad 5995.204 \mathrm{~Hz}$

FIDRES $\quad 0.365918 \mathrm{~Hz}$

$\begin{array}{lc}\text { AQ } & 1.3664756 \mathrm{sec} \\ \text { RG } & 456.1\end{array}$

DW $\quad 83.400$ usec

DE $\quad 6.50$ usec

$01 \quad 150000000$

$\equiv= \pm====$ CHANNEL $f 1 \neq \approx=z===$

$\begin{array}{lll}\text { NUC1 } & 1 \mathrm{H} \\ \mathrm{P} 1 & 10.10 \mathrm{usec}\end{array}$

$\begin{array}{lr}\text { P1 } & 10.10 \text { use } \\ \text { PL1 } & 3.00 \mathrm{~dB}\end{array}$

SF 01 $400.1326000 \mathrm{MHz}$

F2 - Processing parameters

SI 16384

SF $\quad 400.1300091 \mathrm{MHz}$

$\begin{array}{lc}\text { WDW } & \text { EM } \\ \text { SSB } & 0\end{array}$

$\begin{array}{cc}L B & 0.10 \mathrm{~Hz} \\ G B & 0\end{array}$

10 NMR plot parameters

cx $20.00 \mathrm{~cm}$

CY $\quad 8.00 \mathrm{~cm}$

$\begin{array}{ll} & 10.500 \mathrm{ppm}\end{array}$

F2P $\quad-0.500 \mathrm{ppm}$

PPMCM $\quad 0.55000 \mathrm{ppm} / \mathrm{c}$

HZCM $\quad 220.07150 \mathrm{~Hz} / \mathrm{cm}$ 
C13 spectrum of

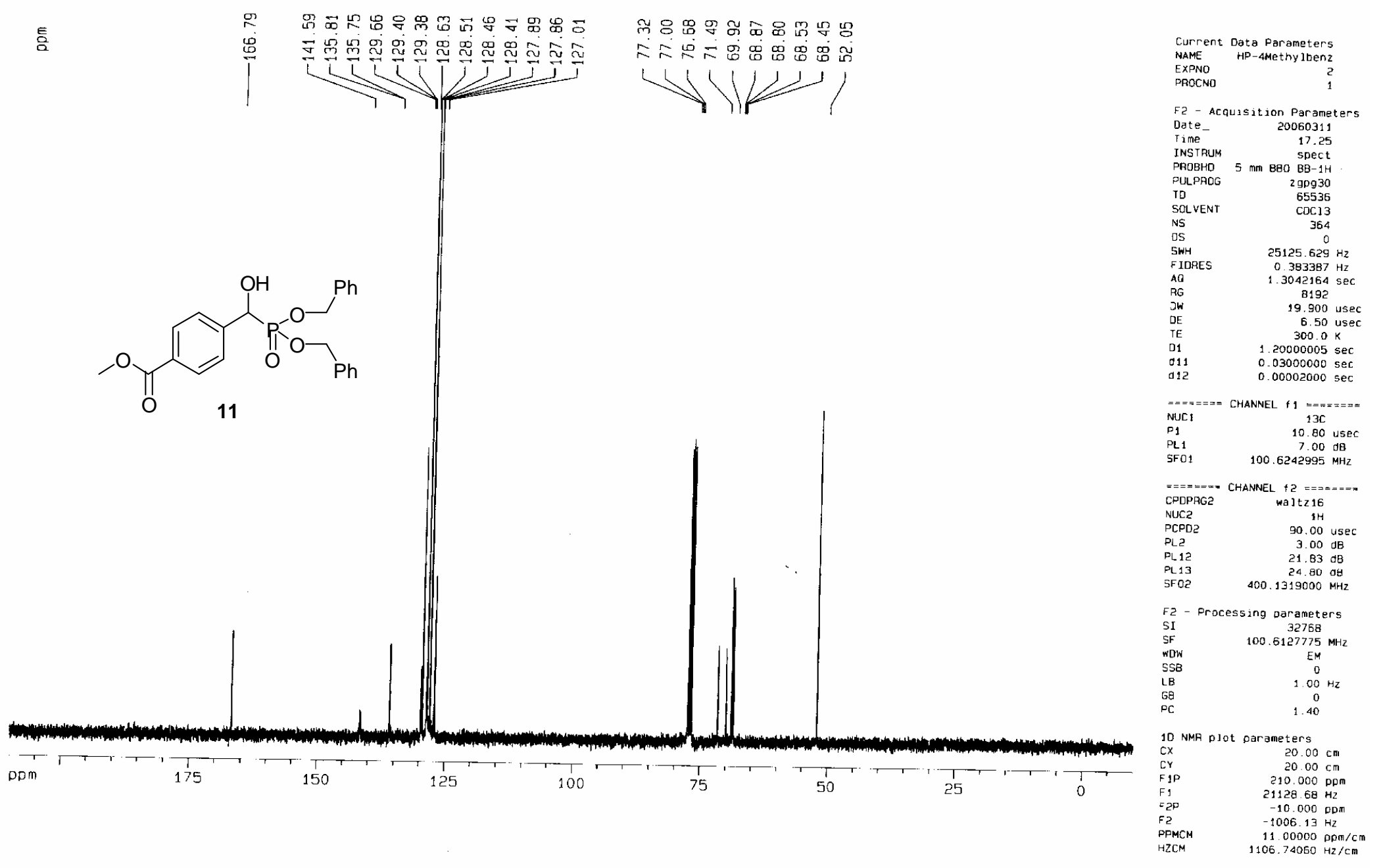


H spectrum o

言

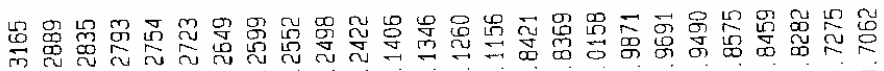

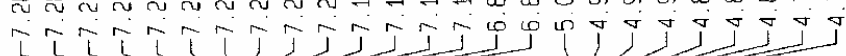

牦

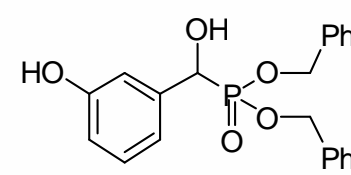

12

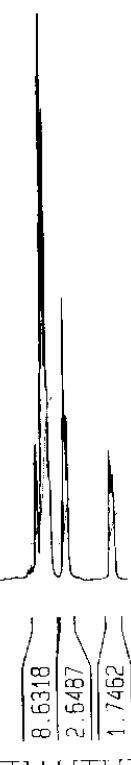

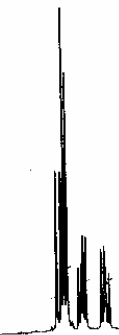

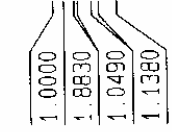

Current Data Parameter

EXPNO

F2 - Acquisition Paramet

Time
INSTRUM

PROBHO $5 \mathrm{~mm}$ BBO BB-1H
PULPROG

16384
TD

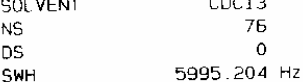

$\begin{array}{ll}\text { SWH } & 5995.204 \mathrm{~Hz} \\ \text { FIDRES } & 0.365918 \mathrm{~Hz}\end{array}$

$\begin{array}{lc}0.365918 \mathrm{~Hz} \\ A Q & 1.3654756 \mathrm{seC} \\ \mathrm{Q} & 256\end{array}$

256
OW

6.50 us
DE
TE
D1

$=z====0$ CHAm 1.50000000

NUC1 $1 \mathrm{H}$

$\begin{array}{lr}\text { P1 } & 10.10 \text { Us } \\ \text { PL1 } & 3.00 \mathrm{~dB}\end{array}$

SF01 400.1326008 MHZ

Fa - Processing parameters

$\begin{array}{lc}\text { SI } & 15384 \\ \text { SF } & 400.1300080 \mathrm{MHZ} \\ \text { WDW } & \text { EM }\end{array}$

$\begin{array}{lc}\text { SSB } & 0 \\ \text { LB } & 0.10 \mathrm{~Hz} \\ \text { SB } & 0\end{array}$

10 NMF olot parameters

$\begin{array}{lr}\text { CX } & 20.00 \mathrm{~cm} \\ C Y & B .00 \mathrm{~cm}\end{array}$

$\begin{array}{lr}\text { Cr } & 8.00 \mathrm{~cm} \\ F 1 P & 10.500 \mathrm{cpm} \\ F 1 & 4201.37 \mathrm{~Hz}\end{array}$

$420.37 \mathrm{~Hz}$
$-0.500 \mathrm{pp}$

$-200.07 \mathrm{~Hz}$

0. $55000 \mathrm{ppm} / \mathrm{cm}$

8

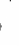

5 
C13 spectrum of

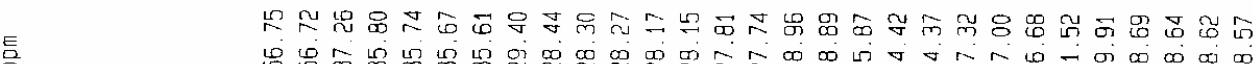

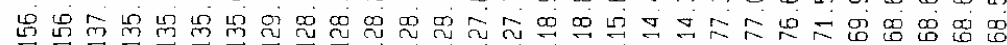
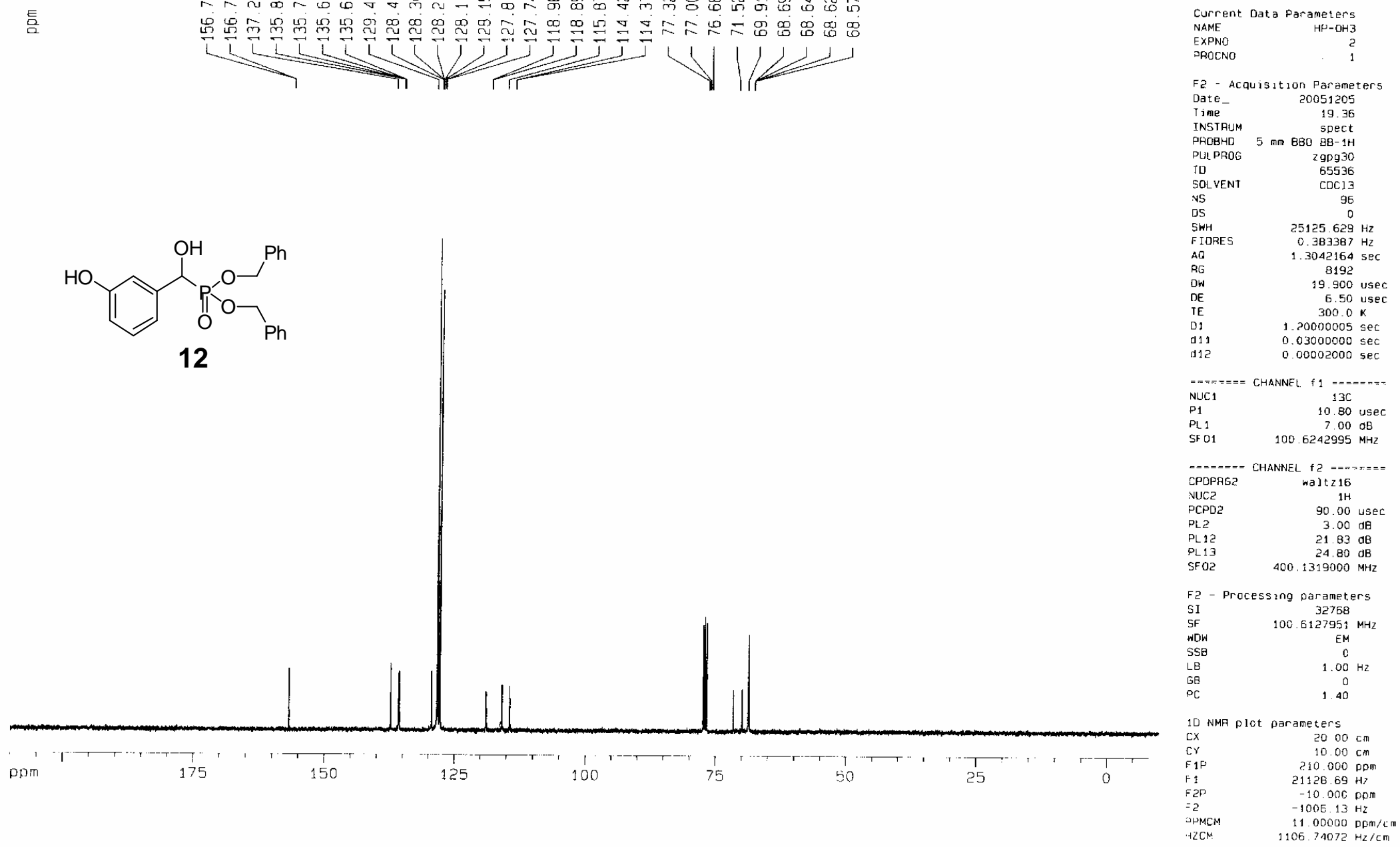
$H$ spectrum of

言

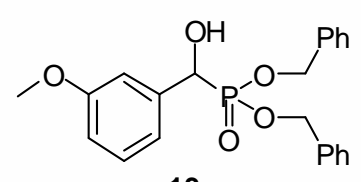

13

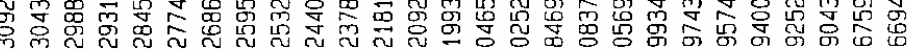
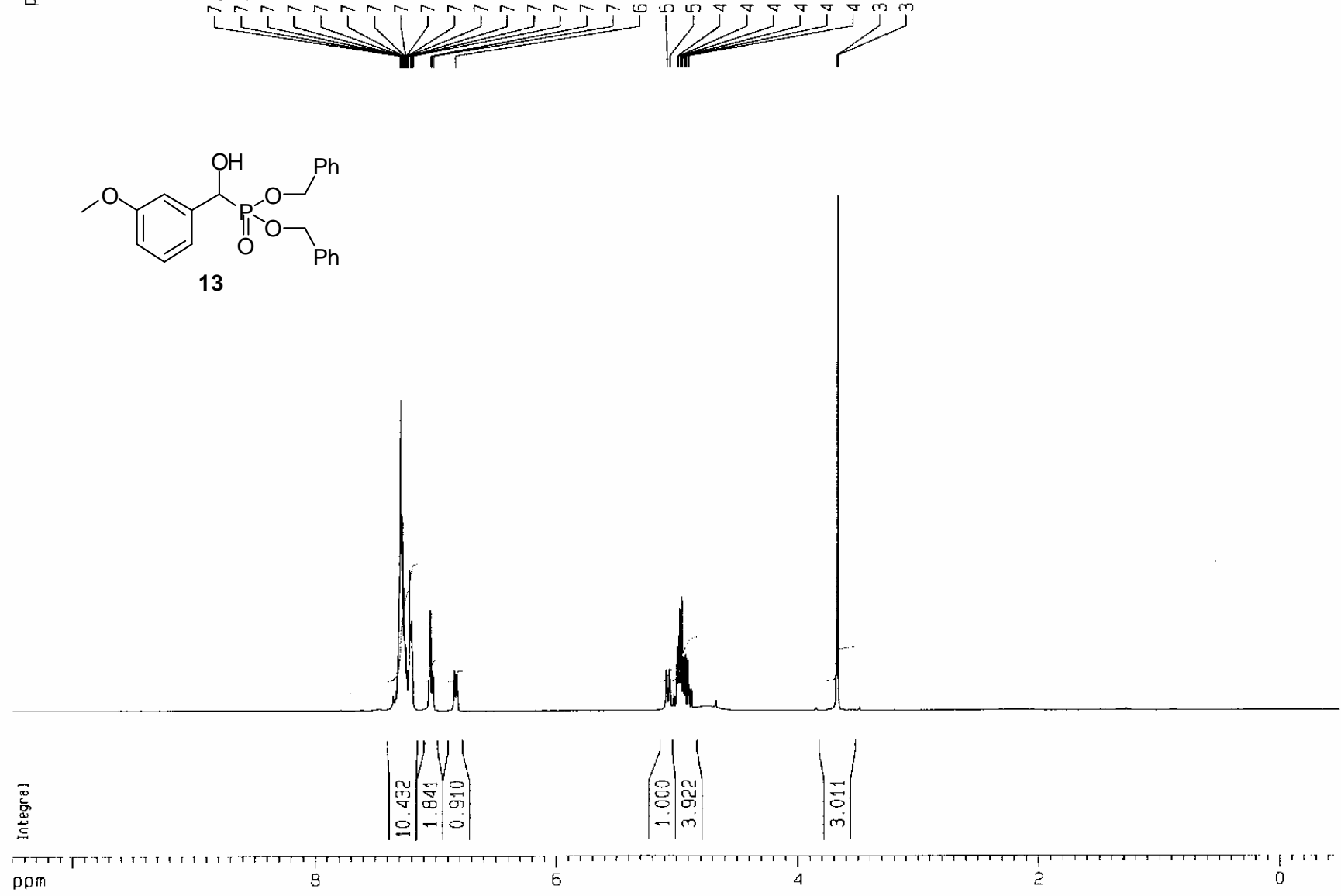

Current Data Parameters

EXPNO

F2 - Acquisition Paramet

Time

$\begin{array}{lr}2930 \\ \text { TD } & 16384 \\ & \end{array}$

$\begin{array}{lr}\text { SOLVENT } & \text { COC13 } \\ \text { NS } & 32\end{array}$

DS $\quad 5995.204 \mathrm{~Hz}$

FIDRES $\quad 0.365918 \mathrm{~Hz}$

$\triangle Q \quad 1.3664756 \mathrm{se}$

$\begin{array}{lr}\text { RG } & 80.6 \\ \text { DW } & 83.400 \text { usec } \\ \text { DE } & 6.50 \text { use }\end{array}$

D1 1.50000005

$=======$ CHANNEL $f 1 \quad======$

$\begin{array}{ll}\text { NUC1 } & 1 \mathrm{H} \\ \mathrm{P} 1 & 10.10 \mathrm{usec}\end{array}$

SFO1 $\quad 3.00 \mathrm{~dB}$

SI 16384

WOW $\quad 400.1300121 \mathrm{M}$

$\begin{array}{lc}\mathrm{SSB} & 0 \\ \mathrm{LB} & 0.10 \mathrm{HZ} \\ \mathrm{GB} & 0\end{array}$

10 NMR plot parameters

CX 20.00

$\begin{array}{lr}\text { CY } & 9.00 \mathrm{~cm} \\ \text { F1P } & 10.500 \mathrm{pom}\end{array}$

$\begin{array}{ll}\text { F1 } & 4201.37 \mathrm{~Hz} \\ \text { F2P } & -0.500 \mathrm{Dom}\end{array}$

$-200.06 \mathrm{~Hz}$

$\begin{array}{ll}\text { PPMCM } & 0.55000 \mathrm{ppm} / \mathrm{cm} \\ \mathrm{HZCM} & 220.07150 \mathrm{~Hz} / \mathrm{cm}\end{array}$ 
C13 spectrum of

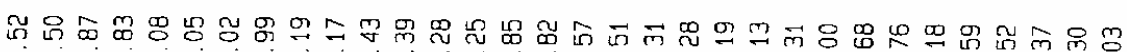

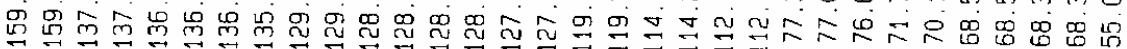

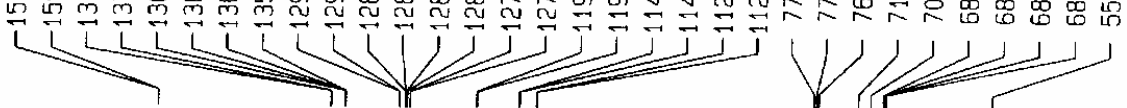

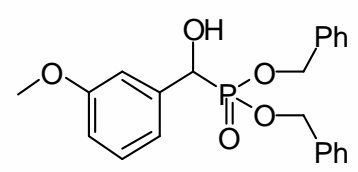

13

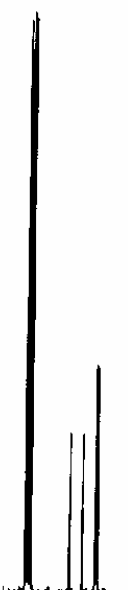

100

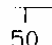

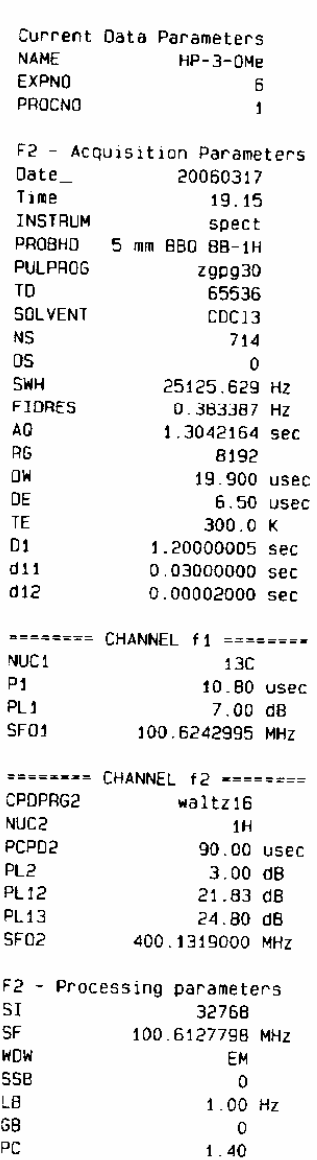

10 NMP plot parameters
$20.00 \mathrm{~cm}$
$12.50 \mathrm{~cm}$

$210.000 \mathrm{ppm}$

$21128.68 \mathrm{~Hz}$

$-10.000 \mathrm{ppm}$
$-1006.13 \mathrm{~Hz}$ $1.00000 \mathrm{pmm} / \mathrm{cm}$ 
$H$ spectrum of

14
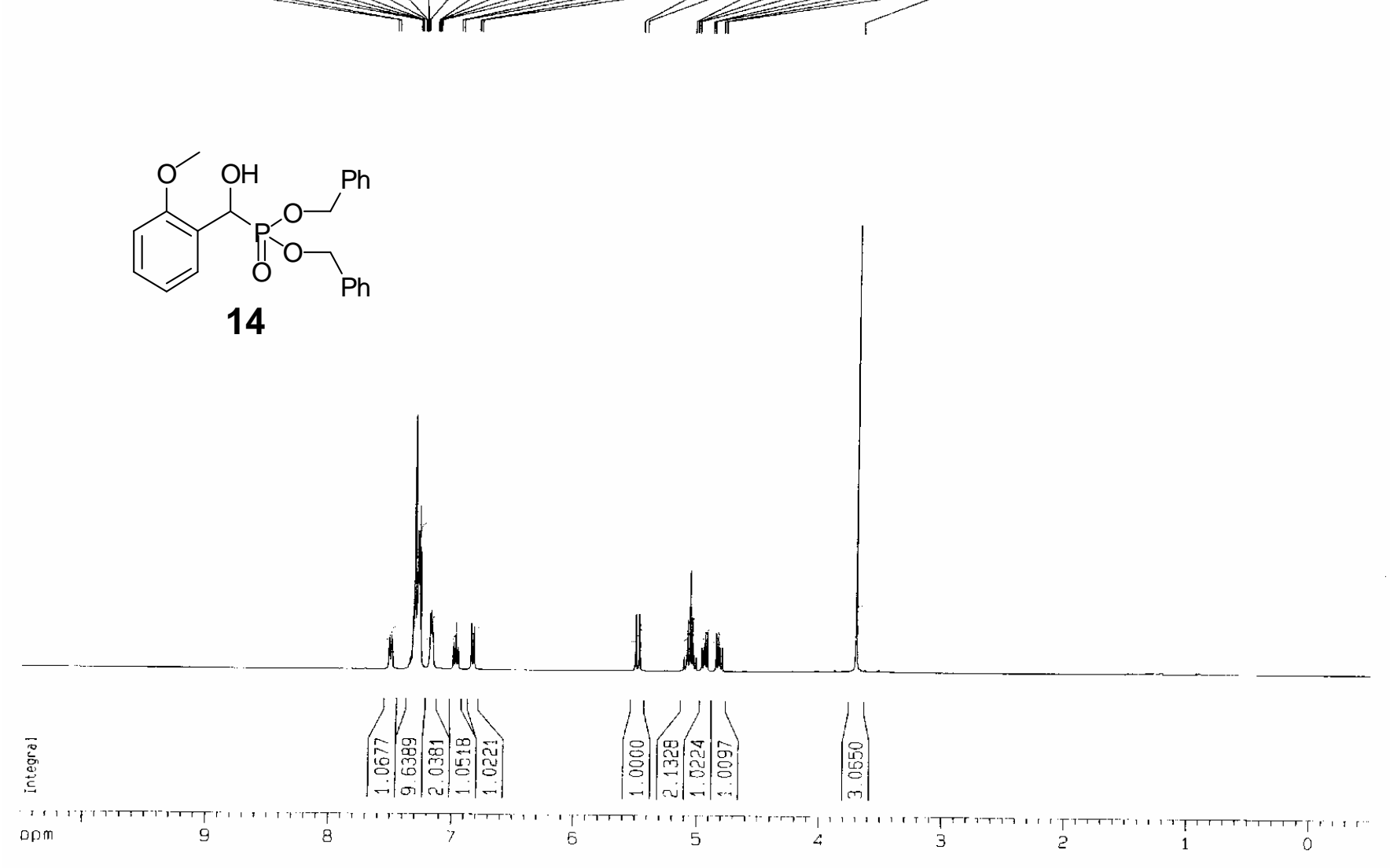

Current Data Parameters
NAME

EXPNO

F2- Acquisition Parameter

Date 20051205

Time

PROBHD $5 \mathrm{~mm} \mathrm{BBO} \mathrm{BDE}-1 \mathrm{H}$

PULPROG 2 zgBO

SOL VENT

NS $\quad 16$

$\begin{array}{ll}\text { SWH } & 5995.204 \mathrm{~Hz}\end{array}$

$\begin{array}{ll}F I D R E S & 0.365918 \mathrm{~Hz} \\ A Q & 1.3654756 \mathrm{sec}\end{array}$

$\begin{array}{rr}A Q & 1.3654756 \mathrm{sec} \\ 26 & 256 \\ D W & 83.400 \text { usec }\end{array}$

DE $\quad 5.50$ use

$01 \quad 15000000 \mathrm{~K}$

$== \pm====$ CHANNEL $i 1$
vUC1 $== \pm= \pm==$

$\begin{array}{ll}\text { NuC1 } & 1 \mathrm{H} \\ \mathrm{P} 1 & 10.10 \mathrm{usec}\end{array}$

$\begin{array}{lr}\text { PL1 } & 3.00 \mathrm{~dB} \\ \text { SFO1 } & 400.1326008 \mathrm{MHz}\end{array}$

F2 - Processing parameters

$\begin{array}{lr}\text { SI } & 16384 \\ 5 F & 400.1300099\end{array}$

WDW $\quad$ EM

558
$1 B$

${ }_{P C}^{G B}$

10 NMR plot parameters

$\begin{array}{lr}20.00 \mathrm{~cm} \\ c \mathrm{cr} & 8.00 \mathrm{~cm} \\ \mathrm{Cr} & 10.500 \mathrm{~cm}\end{array}$

$\begin{array}{ll}F 1 & 10.500 \mathrm{ppm} \\ F & 4201.37 \mathrm{~Hz}\end{array}$

$\begin{array}{ll}F 2 & -0500 \mathrm{pDm} \\ \mathrm{FPMCM} & -200.07 \mathrm{~Hz}\end{array}$

$\begin{array}{ll}\text { PPMCM } & 0.55000 \\ \text { HZCM } & 220.07152 \mathrm{~Hz} / \mathrm{cm}\end{array}$ 
C13 spectrum of

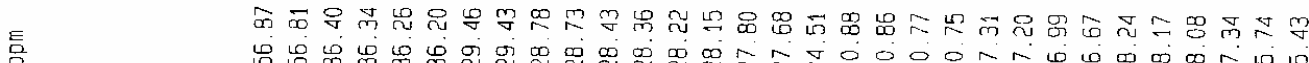

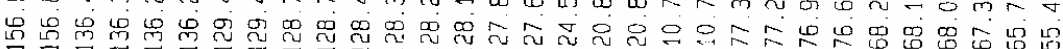

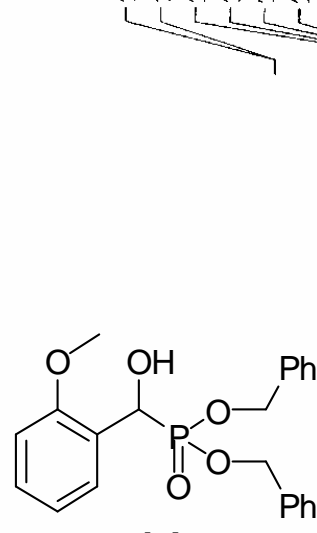

14

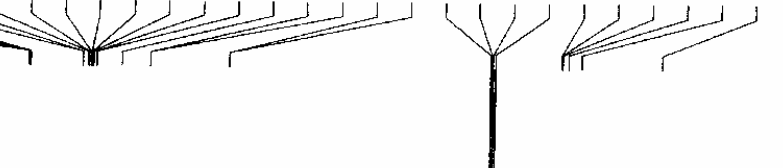

Gurrent Dat a Parameter

NAME

PROCN

iz - Acruisition Parameters
Date- 20051205

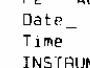

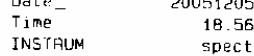

PROBHD $5 \mathrm{~mm} 880 \mathrm{spect}$

290930
ID

DS $\quad 500$

$\begin{array}{ll}\text { SWH } & 25125.629 \mathrm{~Hz} \\ \text { EIDRES } & 0.383387 \mathrm{~Hz}\end{array}$

$\begin{array}{ll}\text { IIDRES } & 0.383387 \mathrm{~Hz} \\ \text { AQ } & 1.30421164 \mathrm{sec} \\ \mathrm{RG} & \end{array}$

$\begin{array}{lr}\text { DW } & 19.900 \text { usec } \\ \text { DE } & 5.50 \text { usec } \\ \text { TE } & 300.0 \text { K }\end{array}$

$011.20000005 \mathrm{sec}$

$\begin{array}{ll}d 11 & 0.03000000 \mathrm{sec} \\ 112 & 0.00002000 \mathrm{sec}\end{array}$

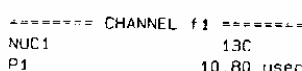

$\begin{array}{lr}P_{1} & 10.80 \text { use } \\ \text { PL1 } & 7.00 \mathrm{~dB} \\ \text { SF01 } & 100.6242995 \mathrm{MHZ}\end{array}$

$====\pi==$ CHANNEL $f 2==== \pm==$
CPDPRGS
NUC?

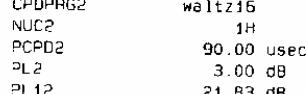

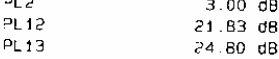

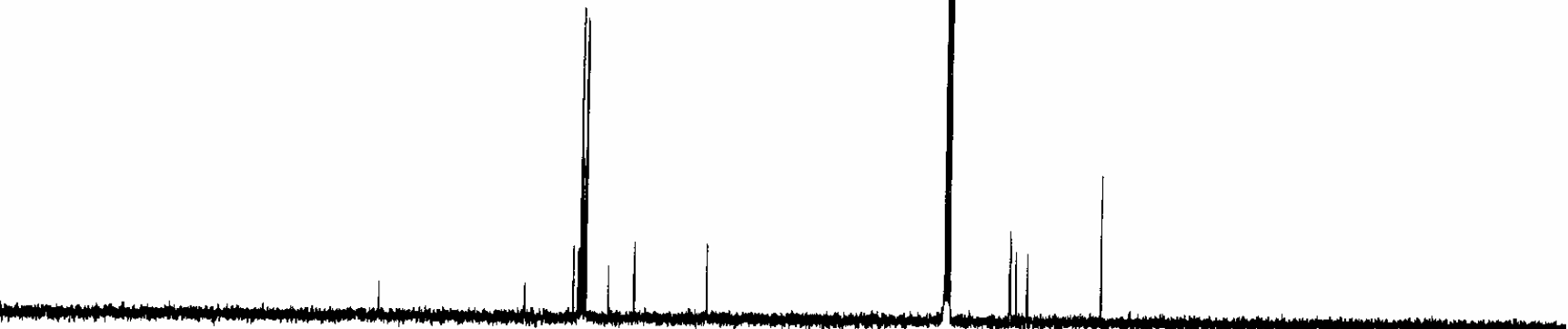

Po

175

150

125

100

$\frac{1}{75}$

$\frac{{ }^{r}-\frac{1}{50}}{}$

25

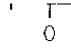

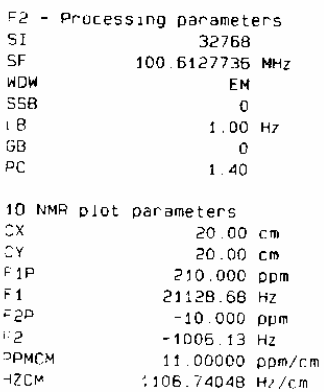


$H$ spectrum of

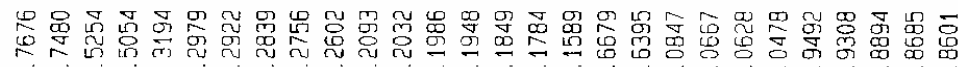

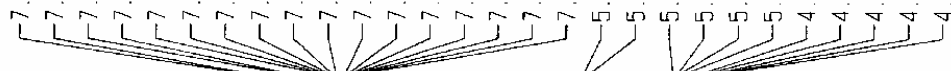
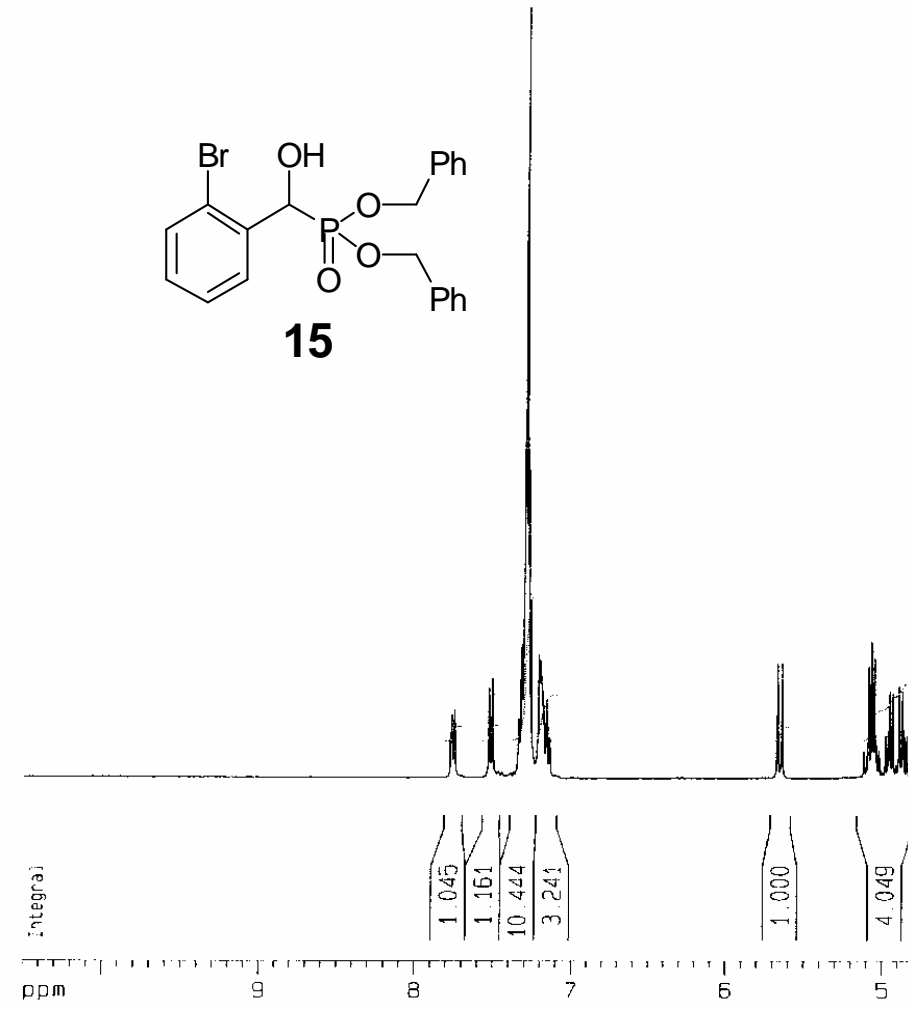

Surrent Data Parameters
VAME

EXPNO

F2 - Acquisition Parameter

Date-

INSTRUM

-10.04
spect

$5 \mathrm{~mm} \mathrm{BBO} B \mathrm{~B}-1 \mathrm{H}$

(1)

SOLVEN

$\begin{array}{lc}\text { NS } & 1 \\ \text { OS } & 0 \\ \text { SWH } & 5995.204 \mathrm{~Hz}\end{array}$

$0.365918 \mathrm{~Hz}$

AQ $\quad 1.3664756 \mathrm{sec}$

$\begin{array}{rr}\mathrm{DW} & 83.400 \mathrm{usec} \\ \mathrm{DE} & 6.50 \mathrm{usec}\end{array}$

$01 \quad 50000000 \mathrm{~K}$

$=z=z= \pm=$ CHANNEL $f 1= \pm= \pm=z=z$

$\begin{array}{lll}\text { NuC1 } & 1 \mathrm{H} \\ \mathrm{D}_{1} & 10.10 \mathrm{usec}\end{array}$

$\begin{array}{lr}\text { P1 } & 10.10 \text { usec } \\ \text { PL1 } & 3.00 \mathrm{~dB} \\ \text { SFO1 } & 400.132500 \mathrm{MHz}\end{array}$

F2 - Processing parameters

$\begin{array}{lr}\text { SI } & 15384 \\ \text { SF } & 400.1300099\end{array}$

WOW 400.1300099 MH

$\begin{array}{lc}\text { SSB } & \text { EM } \\ \mathrm{LB} & 0 \\ \mathrm{CB} & 0.10 \mathrm{~Hz}\end{array}$

$\begin{array}{ll}\mathrm{GB} & \mathrm{OB} \\ \mathrm{PC} & 100\end{array}$

10 NMR Dlot parameters

$\begin{array}{ll}\text { CX } & 20.00 \mathrm{~cm} \\ \text { Cr } & 10.50 \mathrm{~cm}\end{array}$

F1P $\quad 10500 \mathrm{~cm}$

$\begin{array}{ll}\text { F1 } & 10.50 \mathrm{~cm} \\ \text { F2P } & 4201.37 \mathrm{~Hz} \\ \text { F2 } & -0.500 \mathrm{pDm}\end{array}$

$\begin{array}{ll}\text { F2 } & -200.07 \mathrm{~Hz} \\ \text { PPMCM } & 0.55000 \mathrm{pPm} / \mathrm{cm} \\ \text { HZCM } & 220.07152 \mathrm{HZ} / \mathrm{Cm}\end{array}$ 
C13 spectrum of

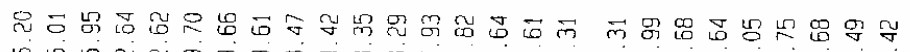

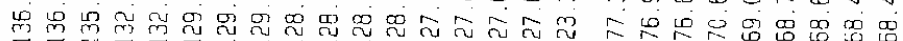

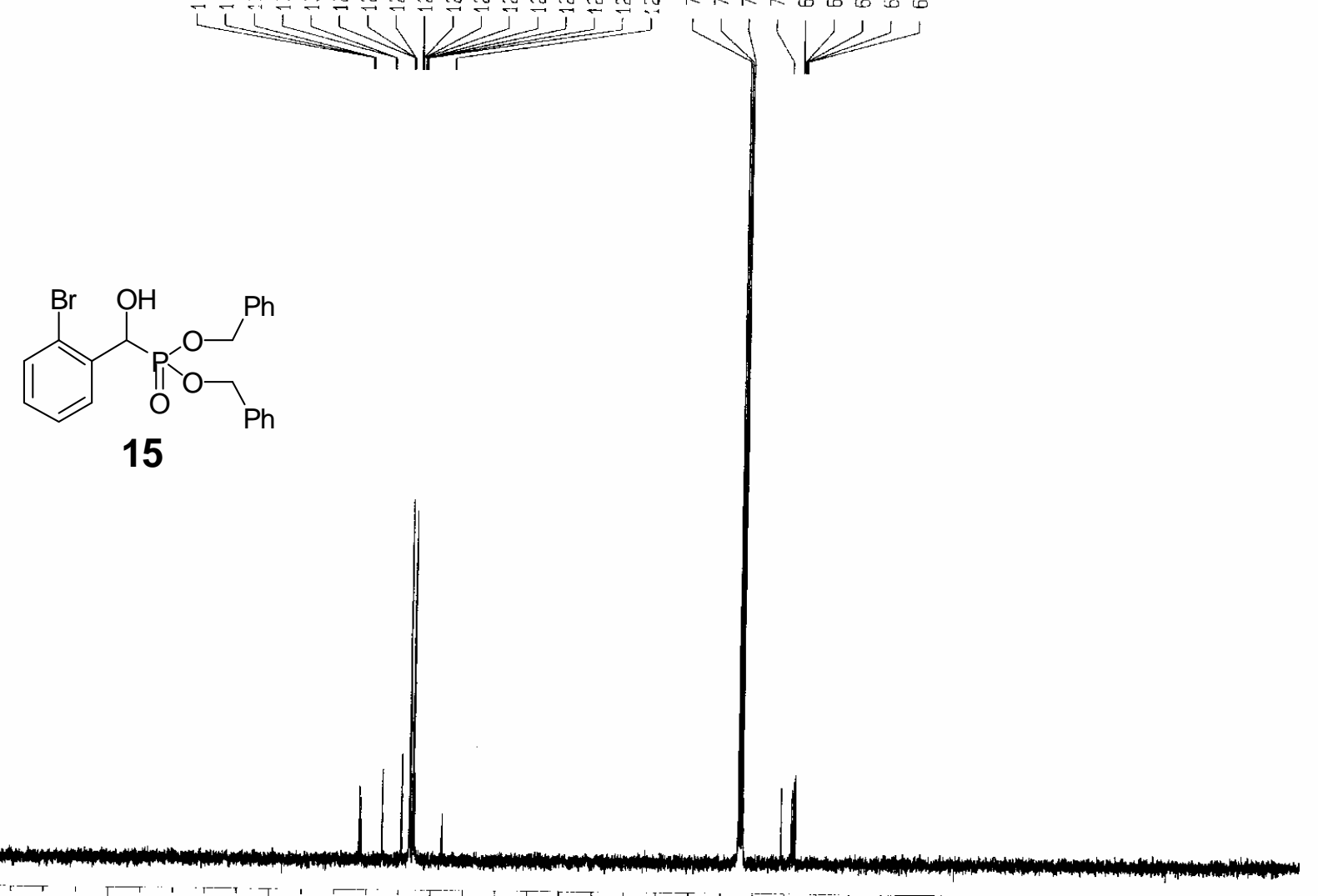

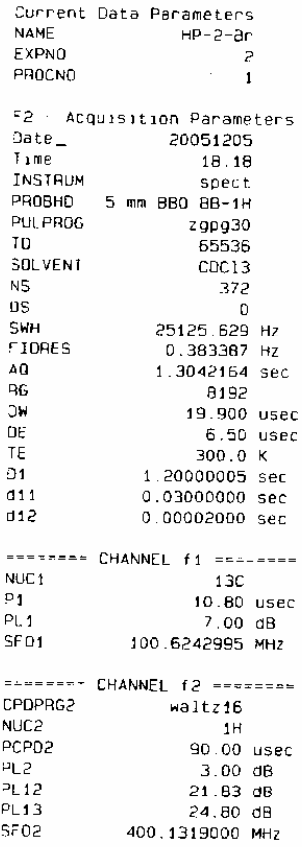

F2 - Processing parameters

Fe Process 1 ng parameters
SI
SF
SF

WOW
SSE
i.

$\mathrm{GB}$
$\mathrm{i} C$

$\log _{1}$

${ }_{175}^{\Gamma} \cdot+\frac{7}{150}$

125

75

$\frac{1}{50}$
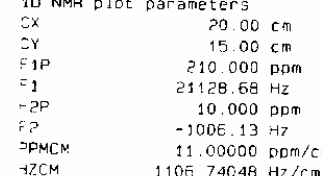
$H$ spectrum of

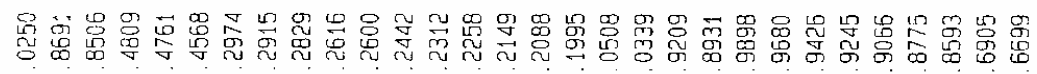

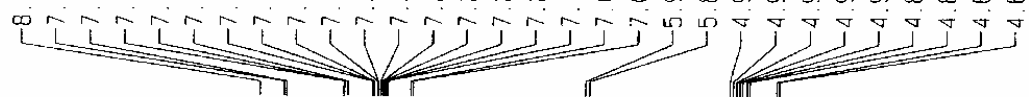

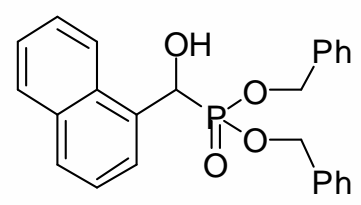

16

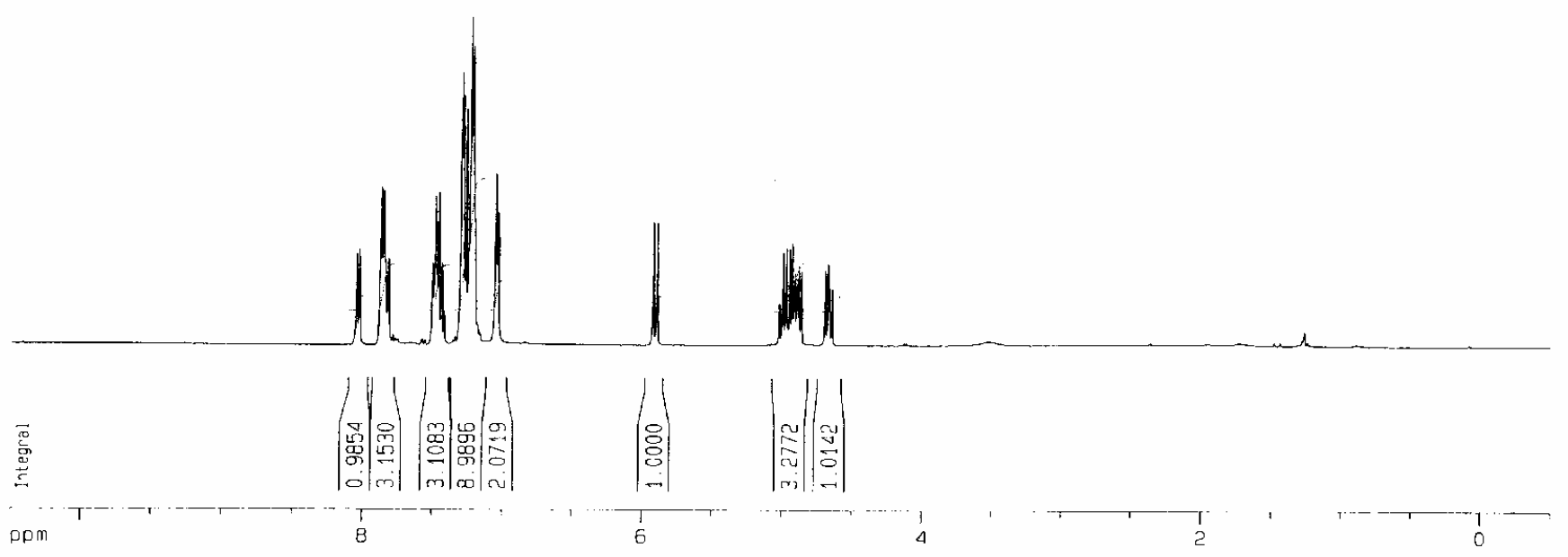

Current Data Parameters
NAME

EXPNO

$=2-4 c q u a s i t i o n$ Paramctors

Date $\quad 20051114$

INST

PROBHID $5 \mathrm{~mm}$ BBO BB-1H

PULPROG 2930

SOI.VENT CDC13
NS

$\begin{array}{lr}\text { NS } & 54 \\ \text { DS } & 0 \\ \text { SWH } & 5995.204 \mathrm{~Hz}\end{array}$

FDRES $\quad 0.365918 \mathrm{~Hz}$

$\begin{array}{rr}A Q & 1.3651756 \mathrm{sec} \\ 26 & 287.4 \\ D W & 83.400 \mathrm{usec}\end{array}$

83.400 usec
6.50 usec

$1.50000000 \mathrm{sec}$

Di

$=== \pm= \pm=$ CHANNEL
NUC1 $1====== \pm$
$11 \mathrm{H}$

P1 10.10 usec

$\begin{array}{ll}3.00 \mathrm{~dB} \\ \text { SF } 01 & 400.1326008 \mathrm{MHz}\end{array}$

F2 - Processing parameters

$\begin{array}{ll}\mathrm{SI} & 16384 \\ \mathrm{SF} & 400.1300084 \mathrm{MHZ}\end{array}$

WDW

IB
GB
$P C$

10 NMB Dlot parameters

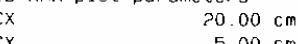

$\begin{array}{lr}\text { F1P } & 15.00 \mathrm{~cm} \\ F 1 & 4201.37 \mathrm{pDm} \\ F & -0.5007\end{array}$

$4201.37 \mathrm{~Hz}$
$-0.500 \mathrm{ppm}$

$\begin{array}{ll}\mathrm{FZ} & -200.07 \mathrm{~Hz} \\ \text { PPMCM } & 0.55000 \mathrm{ppm} / \mathrm{cm} \\ \text { HZCM } & 220.07152 \mathrm{HZ} / \mathrm{Cm}\end{array}$ 
C13 spectrum of

言

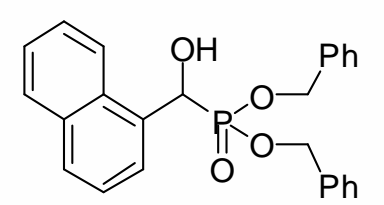

16

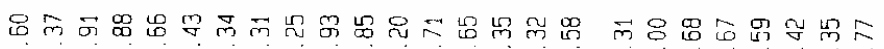

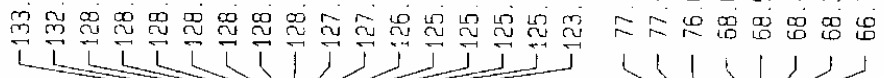

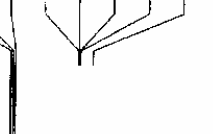

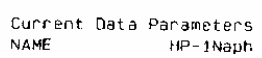

EXPNO

Fe- Acquisition Parameters
Date- 20051205

Iime-
INSTRUM

Spect
PAOBHO

PULPROG
TO

SOLVEN
NS

$\begin{array}{lr}\text { DS } & 0 \\ \text { SWH } & 05125.529\end{array}$

$\begin{array}{ll}25125.629 \mathrm{H} \\ \text { FIDPES } & 0.383387 \\ \mathrm{AO} & \mathrm{Hz}\end{array}$

$\begin{array}{ll}A Q & 1.3042154 \mathrm{sec} \\ \text { RG } & 8192\end{array}$

19.900 usec
OE
TE

$\begin{array}{ll}01 & 1.20000005 \mathrm{sec} \\ \mathrm{d} & 11\end{array}$

$\begin{array}{ll}\text { d11 } & 0.03000000 \mathrm{sec} \\ \mathrm{d} 12 & 0.00002000 \mathrm{sec}\end{array}$

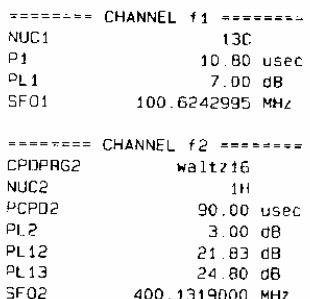

F.- Processing parameters

100.6127736 MHZ

$E M$
0
$1.00 \mathrm{~Hz}$
1.0

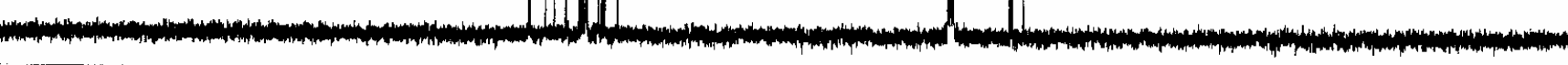

ppm

150

125

100
1
50

\subsection{0}

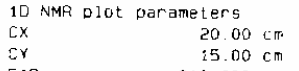

$15.00 \mathrm{~cm}$
$210.000 \mathrm{pmm}$
$21128.68 \mathrm{~Hz}$

$-10.000 \mathrm{pom}$
$-1006.13 \mathrm{H}$ ?

$11.00000 \mathrm{opm} / \mathrm{cm}$
$1106.74048 \mathrm{~Hz} / \mathrm{cm}$ 
$H$ spectrum of

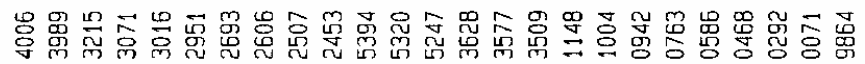
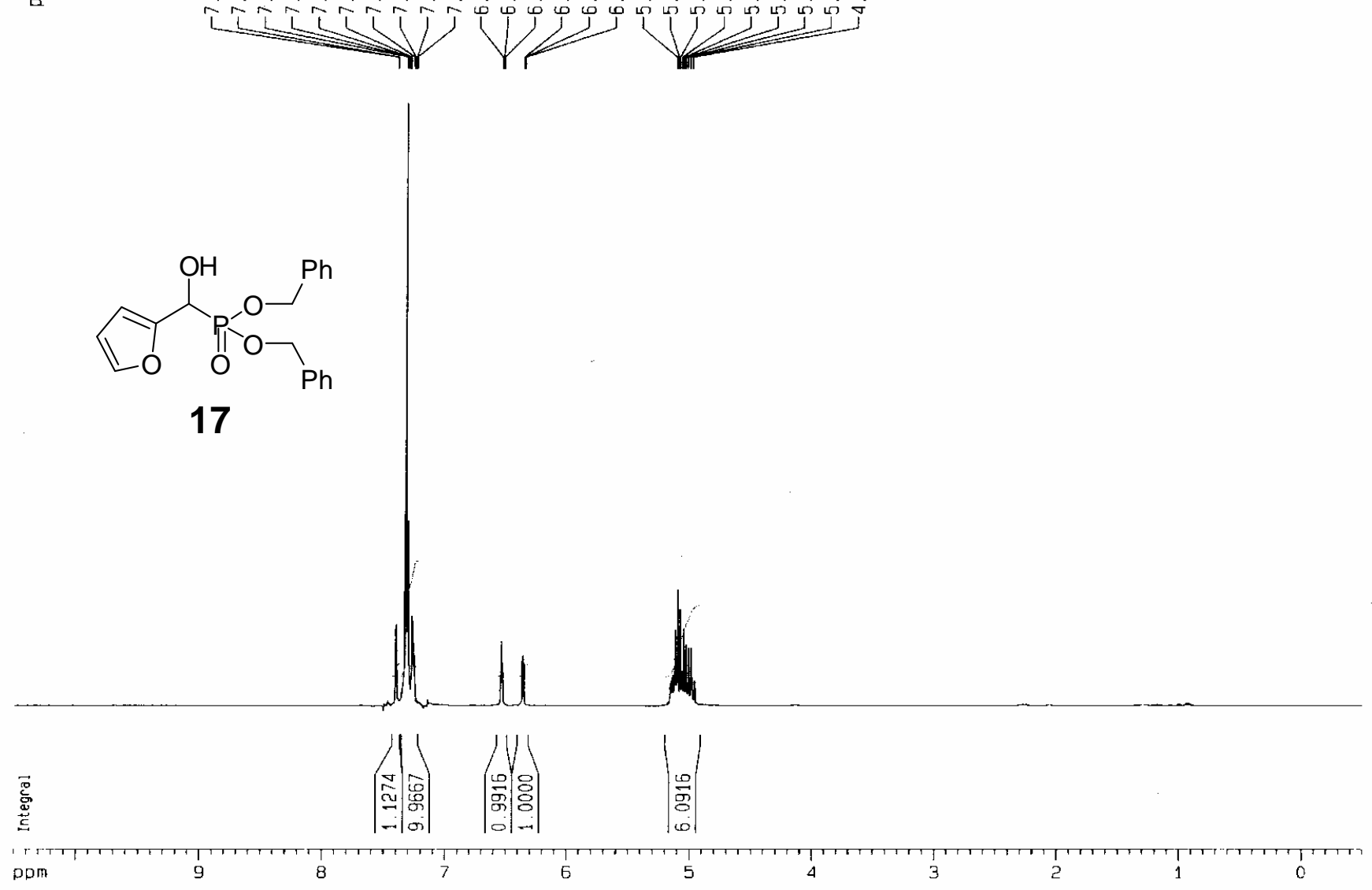

Current Data Parameters
HP-fur

EXPNO

2 - Acquisition Parameters

Time 15.45

2930

SOL VENT $\quad$ CDC13

$\begin{array}{lr}\text { NS } & 1 \\ \text { DS } & 0\end{array}$

$\begin{array}{ll}\text { SWH } & 5995.204 \mathrm{~Hz} \\ \text { FIORES } & 0.365918 \mathrm{~Hz}\end{array}$

AQ $\quad 1.3654756 \mathrm{sec}$

$\begin{array}{lr}\text { DW } & 64 \\ \text { DE } & 83.400 \text { usec }\end{array}$

6.50 use
$300.0 \mathrm{~K}$

$=======$ CHANNEL $f 1=======$
NUC1

Pt1 10.10 user

$3.00 \mathrm{~dB}$
$\mathrm{SF01}$$\quad 400.1326000 \mathrm{MHz}$

F2 - Processing parameters

$\begin{array}{lc}\text { SI } & 16384 \\ \text { SF } & 400.1300069 \mathrm{MHZ}\end{array}$

SSB

$\mathrm{LB}$
$\mathrm{GB}$
$\mathrm{PC}$

1.00

10 NMR Dlot parameters

$\begin{array}{lr}\text { CY } & 20.00 \mathrm{~cm} \\ \mathrm{CY} & 10.50 \mathrm{~cm} \\ \mathrm{~F} & 10.500 \mathrm{~cm}\end{array}$

$\begin{array}{ll}F 1 & 10.500 \mathrm{pDm} \\ F 1 & 4201.37 \mathrm{~Hz}\end{array}$

F2 $-200.07 \mathrm{~Hz}$

$\begin{array}{ll}\mathrm{PPMCM} & 0.55000 \mathrm{ppm} / \mathrm{cm} \\ \mathrm{HZCM} & 220.07152 \mathrm{~Hz} / \mathrm{cm}\end{array}$ 
C13 spectrum of

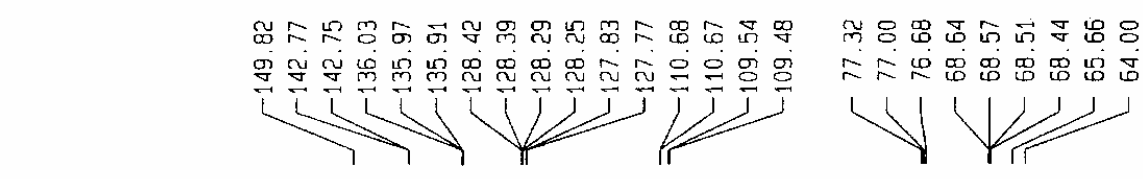

Current Data Parameters

EXPNO

2 - Acquisition Parameters

Date_

Time
INSTRUM

PULPRO $5 \mathrm{~mm}$ B日O BQ-1H

$\begin{array}{lr}\text { TD } & 65536 \\ \text { SOLVENT } & \text { CDC } 13\end{array}$

$\begin{array}{lr}\text { DS } & 0 \\ \text { SHH } & 25125.629 \mathrm{~Hz} \\ \text { FHDRES } & 0.383837 \mathrm{~Hz}\end{array}$

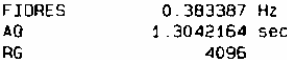

$\begin{array}{lr}\text { RG } & 4096 \\ \text { DW } & 19.900 \text { usec } \\ \text { DE } & 6.50 \text { use } \\ \text { TE } & \text { S.50 }\end{array}$

$\begin{array}{lr}\text { IE } & 300.0 \mathrm{~K} \\ \text { D1 } & 1.20000005 \mathrm{sec}\end{array}$

$\begin{array}{ll}\text { D1 } & 1.20000005 \mathrm{sec} \\ 011 & 0.0300000 \mathrm{sec} \\ 012 & 0.00002000 \mathrm{sec}\end{array}$

$\underbrace{}_{0}{ }_{\mathrm{O}}^{\mathrm{O}}$

17

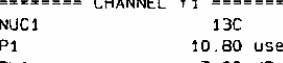

$\begin{array}{lr}P L 1 & 7.00 \text { oB } \\ \text { SF } & 100.6242995 \\ \text { MHZ }\end{array}$

$====x==$ CHANNEL $f 2==x===-$
CPDPRG?

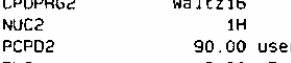

$\begin{array}{ll}\text { PL2 } & 3.00 \mathrm{oB} \\ \text { PL12 } & 21.83 \mathrm{oB}\end{array}$

$\begin{aligned} \text { PL13 } & \begin{aligned} 24.80 \\ \text { PFOB }\end{aligned} \\ \text { SF } & 400.1319000 \mathrm{MHZ}\end{aligned}$

F2 - Processing parameters

SI Processing parameters

$\begin{array}{lc}\text { SF } & 100.6127813 \mathrm{MHz} \\ \text { WOW } & \mathrm{EM} \\ \text { SSB } & 0\end{array}$

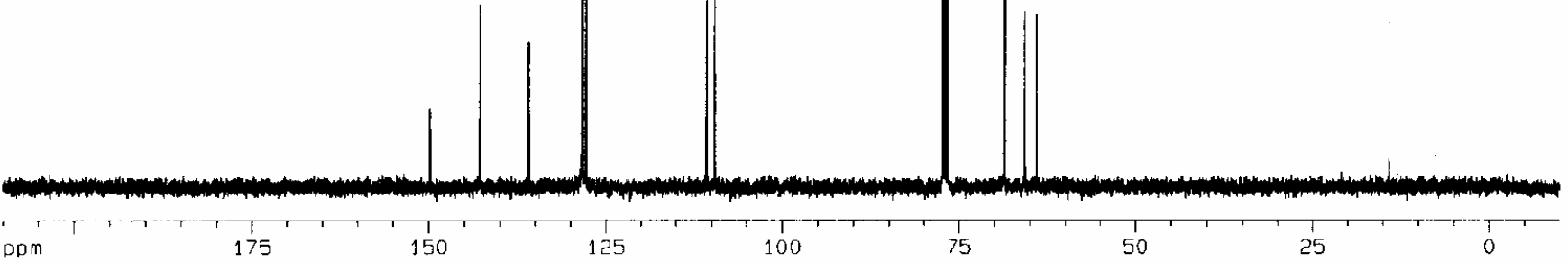

$\underset{P C}{69}$

10 NMP pjot parameters
CX $20.00 \mathrm{~cm}$

$\begin{array}{lr}\text { CX } & 20.00 \mathrm{~cm} \\ \mathrm{CY} & 12.50 \mathrm{~cm} \\ \mathrm{FH} & 210.000 \mathrm{ppm} \\ \mathrm{F} 1 & 21128.68 \mathrm{~Hz}\end{array}$

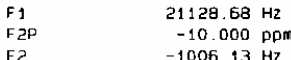

$\begin{array}{ll}\mathrm{F} 2 \mathrm{P} & -10.000 \mathrm{ppm} \\ \mathrm{F2} & -100.13 \mathrm{~Hz} \\ \text { PPMCM } & 11.00000 \mathrm{ppm} / \mathrm{cm} \\ \mathrm{HZCM} & 1106.72008 \mathrm{~Hz} / \mathrm{cm}\end{array}$ 
$H$ spectrum of

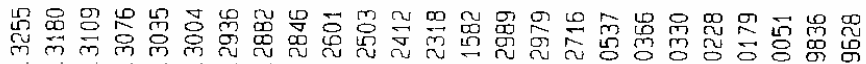
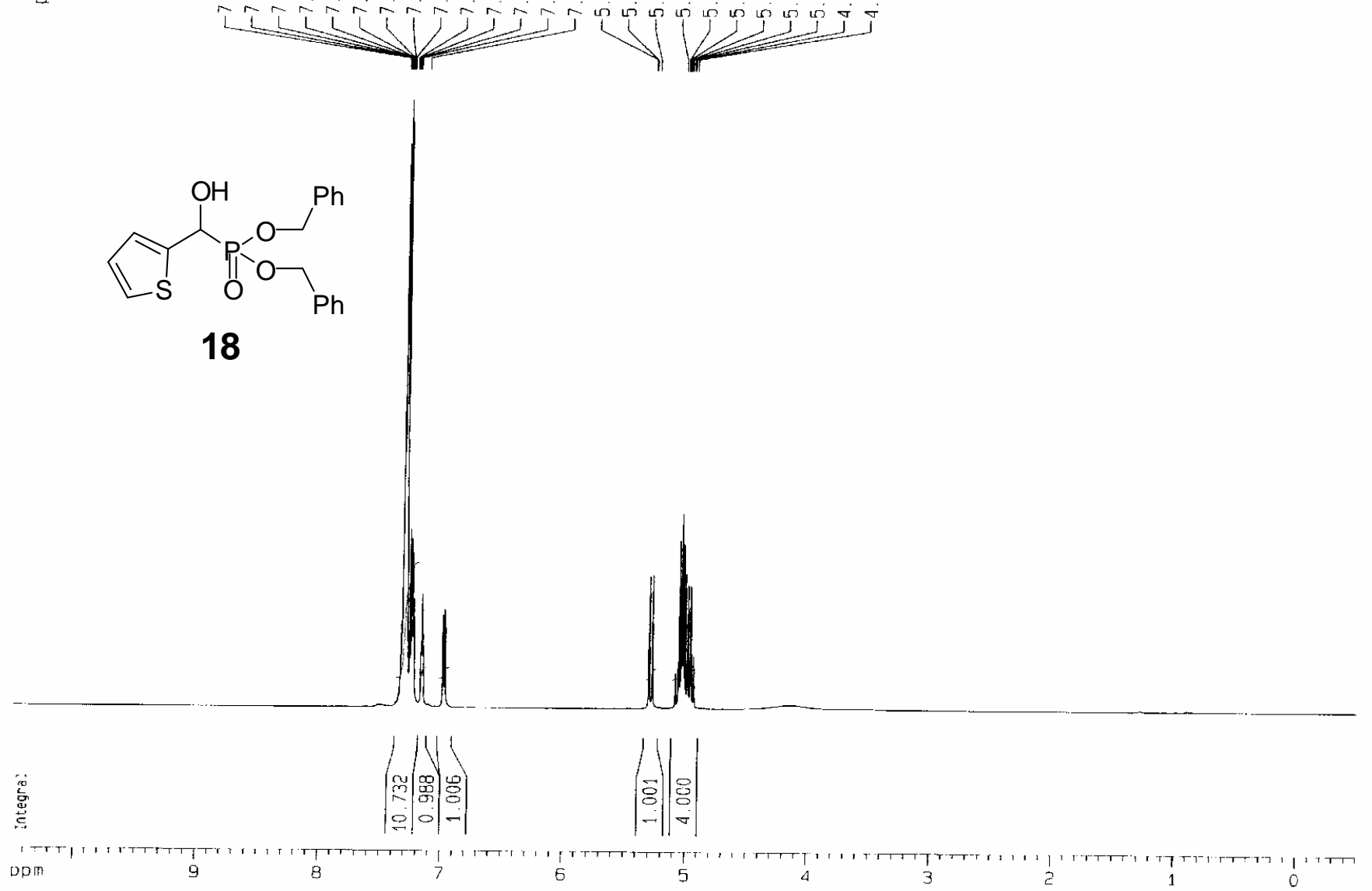

Current Data Parameters

EXPNO 1

2 - Acquisition Parameter

Date
Dacquis
Datio

Time

14.36
spect

SOLVEN

OS

FIDRES $\quad 0.365918 \mathrm{~Hz}$

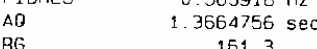

DW 83.400 usec

IE

1.50000000 set

NUC1 $== \pm=$ CHANNEL $f 1==+z===$

$\begin{array}{ll}1 \mathrm{H} & 1 \mathrm{H} \\ \mathrm{P}_{1} & 10.10 \mathrm{usec}\end{array}$

$\begin{array}{rr}3.00 \mathrm{~dB} \\ \text { SF 01 } & 400.1326008 \mathrm{MHZZ}\end{array}$

F2 - Processing parameters

SI 16384

WOW $4001300099 \mathrm{MHz}$

$G B$

D NMR plot parameters

$\begin{array}{ll}C X & 20.00 \mathrm{~cm} \\ C Y & 10.50 \mathrm{~cm}\end{array}$

$\begin{array}{lr}\mathrm{F}_{1 P} & 10.50 \mathrm{~cm} \\ \mathrm{C}_{1} & 10.500 \mathrm{pDm}\end{array}$

$4201.37 \mathrm{~Hz}$
$-0.500 \mathrm{pom}$

$-0.500 \mathrm{ppm}$
$-200007 \mathrm{~Hz}$

$0.55000 \mathrm{ppm} / \mathrm{cm}$
$220.07152 \mathrm{~Hz} / \mathrm{cm}$ 
C13 spectrum of
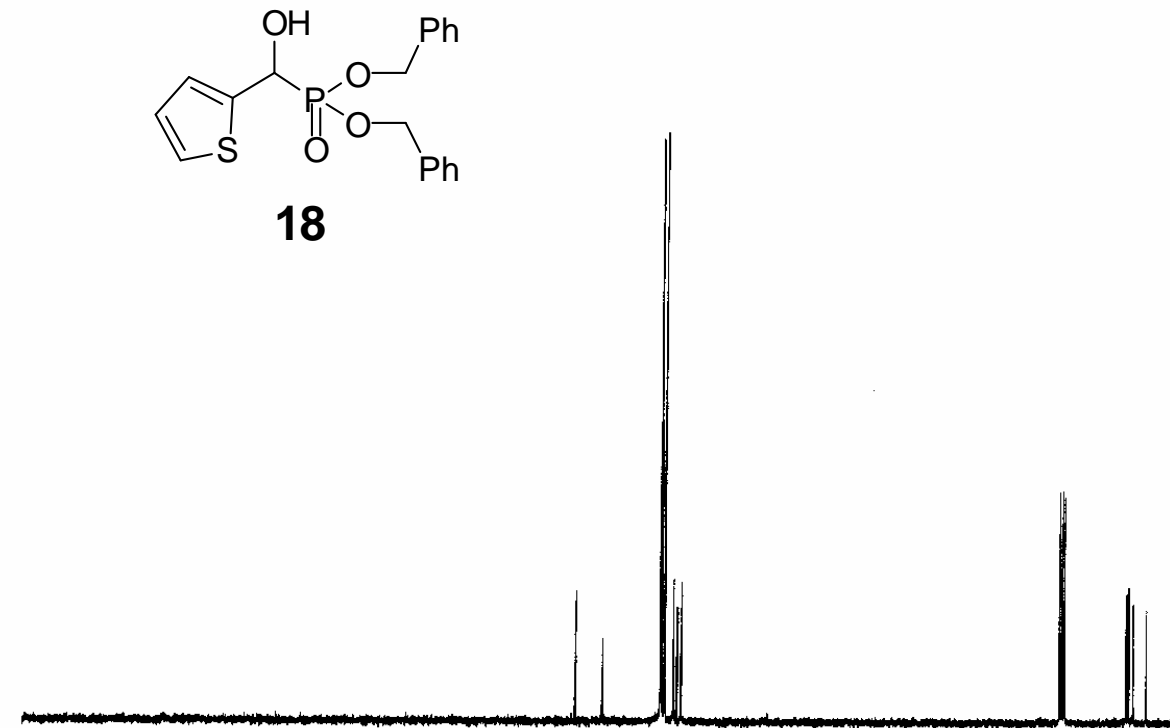

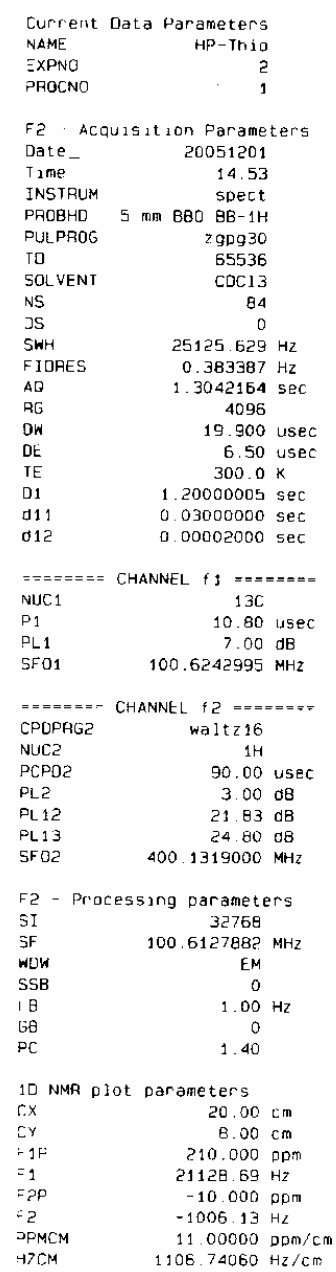


$H$ spectrum of

言

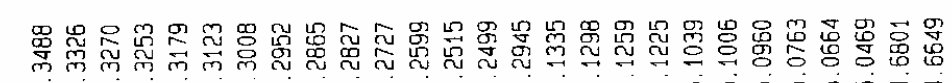

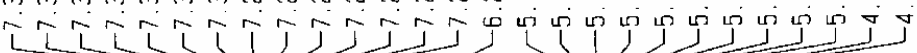

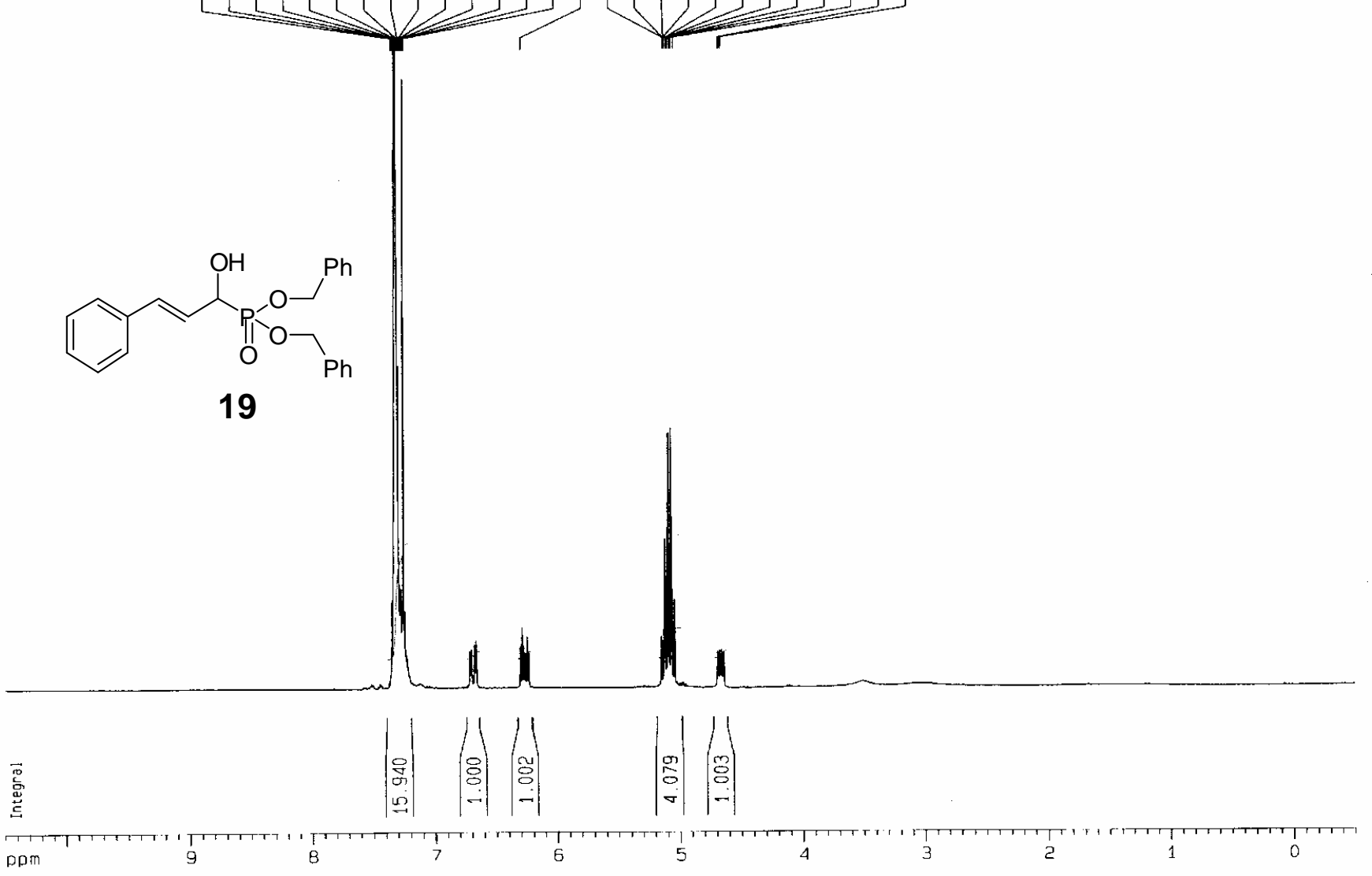

Current Data Parameters
HP-Cin

EXPNO

F2 - Acquisition Parameter

INSTRUM
spect

$\begin{array}{lr}\text { PULPROG } & 2930 \\ \text { TD } & 16384\end{array}$

SOLVENT COC13

NS

SWH $\quad 5995.204 \mathrm{~Hz}$

FARES $\quad 0.365918 \mathrm{~Hz}$

AQ $\quad 4.3654756 \mathrm{sec}$

83.400 use
JE

$1.50000000 \mathrm{k}$

$== \pm==x=$ CHANNEL $f 1=x=====$
NUC1
PUt

$\begin{array}{lll}P_{1} & 10.10 \text { use }\end{array}$

$\begin{array}{ll}3.00 \mathrm{~dB} \\ \text { SFO1 } & 400.1326008 \mathrm{MHZ}\end{array}$

F2 - Processing parameters

$\begin{array}{ll}\text { SI } & 16384 \\ \text { SF } & 400.1300091 \\ \text { MHIZ }\end{array}$

$\begin{array}{cc}\text { SF } & 400.1300091 \\ \text { WDW } & \text { EM } \\ \text { SSB } & 0\end{array}$

$L B$
$G B$
$P C$

10 NMA plot parameters
CX $20.00 \mathrm{~cm}$

$\begin{array}{lr}\text { CX } & 20.00 \mathrm{~cm} \\ \mathrm{CY} & 15.00 \mathrm{~cm} \\ \mathrm{~F} & 10.500 \mathrm{~cm}\end{array}$

$\begin{array}{lr}F_{10} & 10.500 \mathrm{pDm} \\ F_{1} & 4201.37 \mathrm{~Hz} \\ F_{2 P} & -0.500 \mathrm{ppm}\end{array}$

F2 $-200.07 \mathrm{~Hz}$

$\begin{array}{ll}\text { PPMCM } & 0.55000 \mathrm{pDm} / \mathrm{cm} \\ \mathrm{HZCM} & 220.07152 \mathrm{~Hz} / \mathrm{cm}\end{array}$ 
13 spectrum of

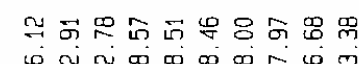

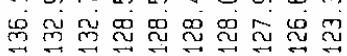
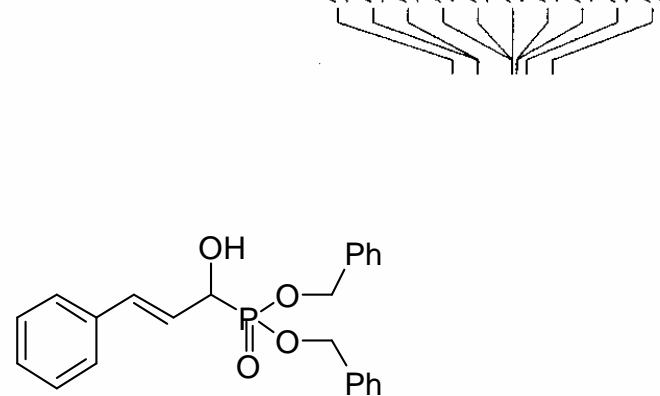

19
요욤ㅁำ

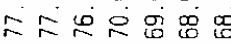

W1 $\begin{array}{lr}\text { CUrrent Data Parameters } \\ \text { NAME } \\ \text { EXPNO } \\ \text { PROCNO } & 2 \\ \text { PROCNO } & 1\end{array}$

F2 - ACQu15ition Parameters
Date- 20051111
Iing

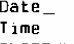

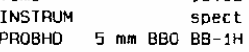

PPobBO
PuLPROL

TD

Solv
NS

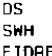

$\begin{array}{ll}A 0 & 1.3042164 \mathrm{sec} \\ \text { AG } & 10.390 \mathrm{wec}\end{array}$

6502 use
OW
DE
DE

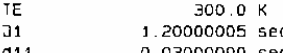

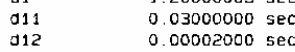

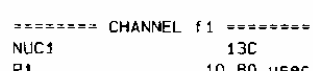

$\begin{array}{ll}\text { NuC9 } & 13 \mathrm{CO} \\ \text { No } & 10.80 \mathrm{usec} \\ \text { Ph } & 7.00 \mathrm{~dB}\end{array}$

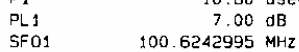

Nan...- CHANNEL $f 2== \pm== \pm=$
CPDPRG
waltz16

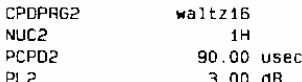

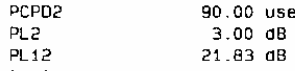

PL12
PL13
PFo
SFo

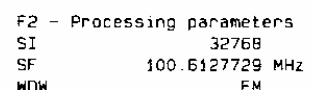

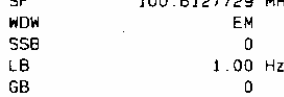

$G B$
$P C$

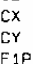

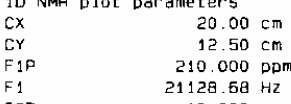

$\begin{array}{ll}\text { F2 } & -10.000 \mathrm{opm} \\ \text { PPMCM } & -1006.13 \mathrm{~Hz}\end{array}$ ppm
1
50 

$\overbrace{\mathrm{Ph}}^{\mathrm{Ph}}$

20

\section{4 spectrum of}

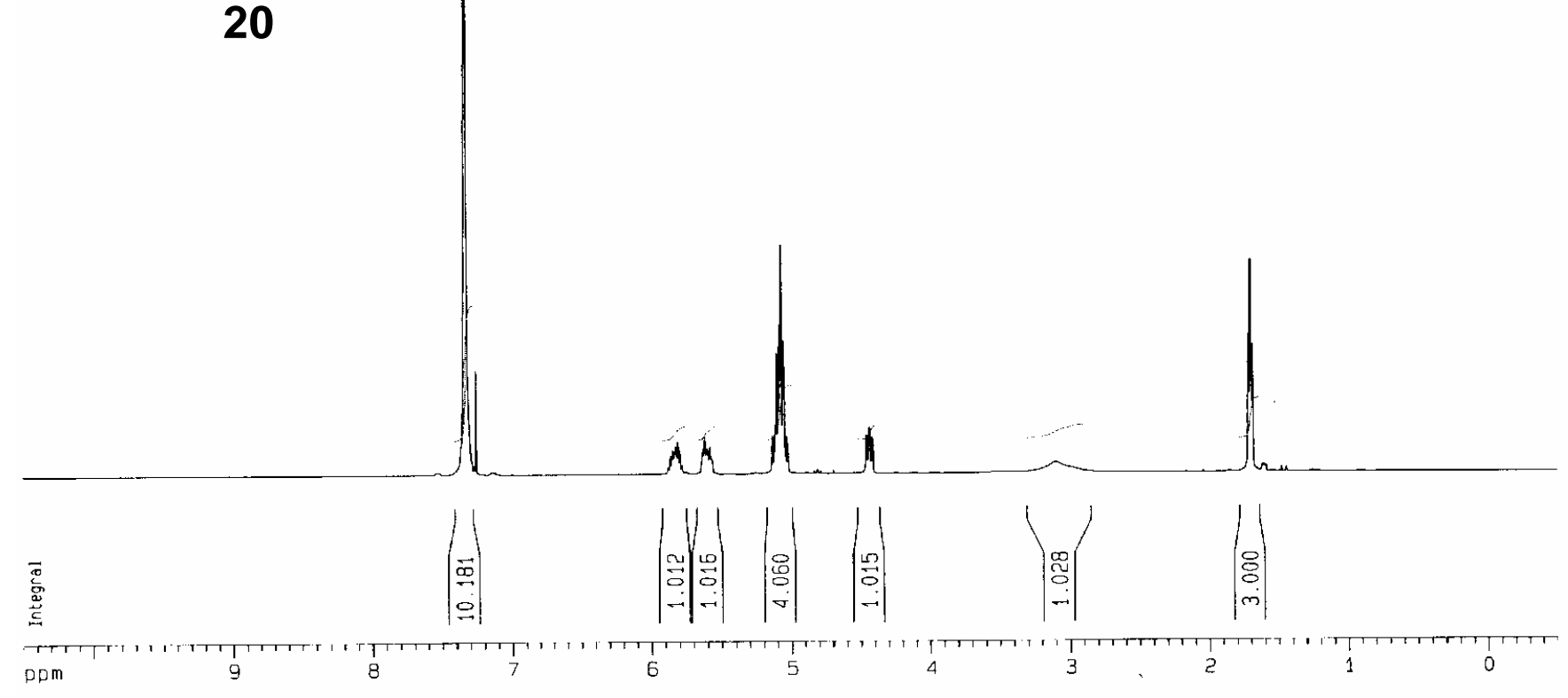

Current Data Parameters

NAME HP-Crot-phos

EXPNO

F2 - Acquisition Parameters

Date_ 20060117

Time
Tinstpuy
Date

INSTRUM spect

PROBHD $5 \mathrm{~mm}$ BBO BB-1H

PULPPOO
TD

SOLVENT

$\mathrm{zg} 30$
16384

NS $\quad 32$

$\begin{array}{ll}\text { DS } & 0 \\ \text { SWH } & 5995.204 \mathrm{~Hz}\end{array}$

AQ $\quad 1.365918 \mathrm{~Hz}$

$\begin{array}{lc}\text { AQ } & 1.3664756 \mathrm{sec} \\ \text { RG } & 143.7\end{array}$

OW
DE

$\begin{array}{cc}\text { DE } & 5.50 \text { use } \\ \text { TE } & 300.0 \mathrm{~K} \\ \text { D1 } & 1.500000005\end{array}$

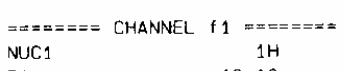

$\begin{array}{ll}\text { NUC1 } & 1 \mathrm{H} \\ \text { P1 } & 10.10 \mathrm{usec}\end{array}$

$3.00 \mathrm{~dB}$
$\mathrm{SFO} 01$

F2 - Processing parameters

SI 16384

WFW $\quad 400.1300095 \mathrm{M}$

$\begin{array}{lc}\text { SSB } & 0 \\ L B & 0.10 \mathrm{~Hz}\end{array}$

$\begin{array}{lr}\mathrm{GB} & 0 \\ \mathrm{PC} & 1.00\end{array}$

10 NMR plot parameters
20.00

$\begin{array}{ll}C X & 20.00 \mathrm{~cm} \\ \mathrm{Cr} & 20.00 \mathrm{~cm}\end{array}$

$\begin{array}{ll}=1 \mathrm{P} & 10.500 \mathrm{ppm} \\ =1 & 4201.37 \mathrm{~Hz}\end{array}$

$4201.37 \mathrm{~Hz}$

$\begin{array}{ll}F & -0.500 \mathrm{ppm} \\ F 2 & -200.07 \mathrm{~Hz} \\ \text { PPMCM } & 0.55000 \mathrm{ppm} / \mathrm{cm}\end{array}$

$\begin{array}{lr}\text { PPMCM } & 0.55000 \mathrm{ppm} / \mathrm{cm} \\ \text { HZCM } & 220.07152 \mathrm{~Hz} / \mathrm{cm}\end{array}$ 
C13 spectrum of
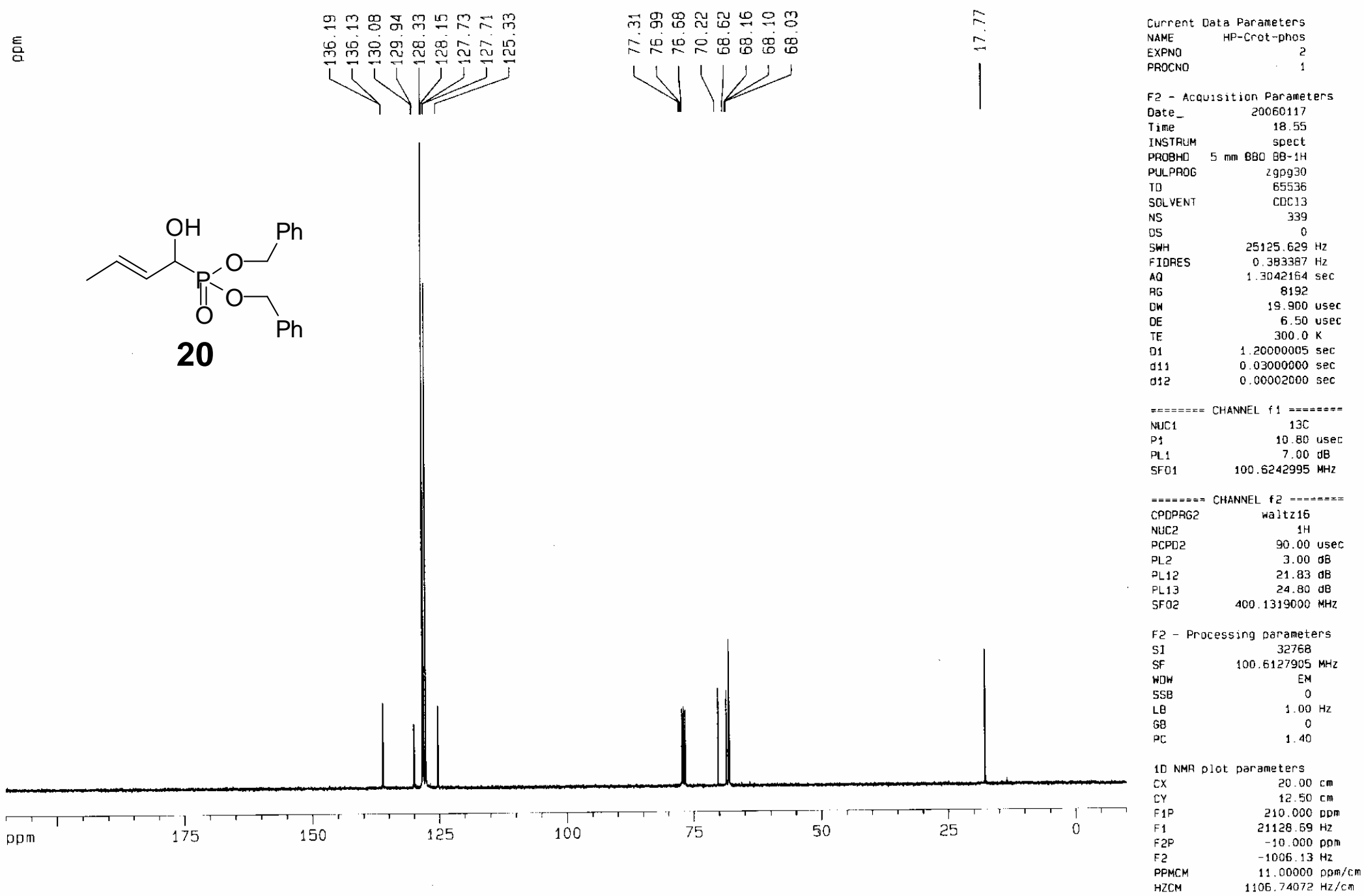
$H$ spectrum of

言

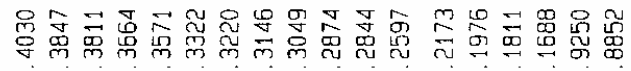

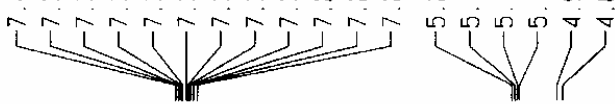

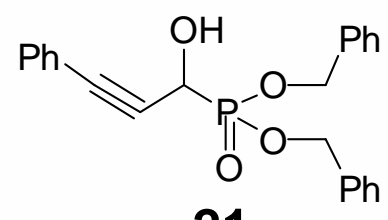

21

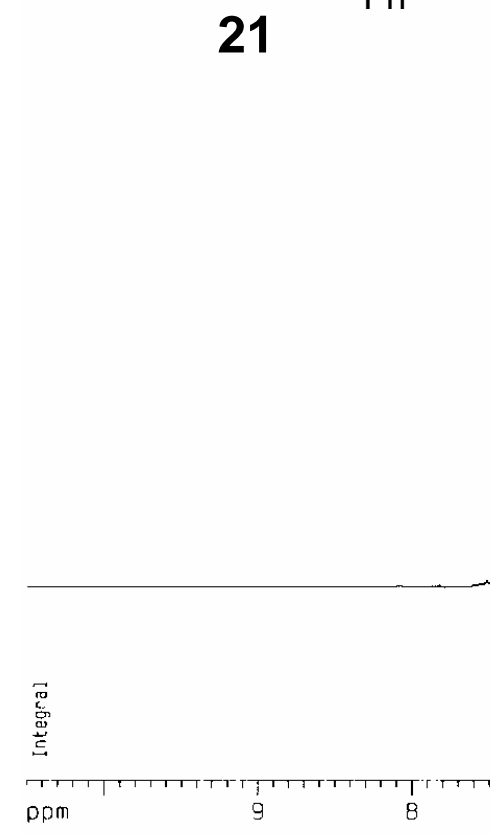

Current Data Parameters

NAME HP-ph-prop

PROCNO

F2 - Acquisition Parameters

Date 20060120

INSTRUM

PROBHD $5 \mathrm{~mm}$ BBO $\mathrm{BB}-1 \mathrm{H}$

PULPAOG 2930

$\begin{array}{ll}\text { ID } & 16384 \\ \text { SOLVENT } & \text { COCI3 }\end{array}$

NS 100

SWH $\quad 5995.204 \mathrm{~Hz}$

FIDRES $\quad 0.365918 \mathrm{~Hz}$

$\begin{array}{lc}A Q & 1.3664756 \mathrm{sec} \\ \mathrm{AQ} & 512\end{array}$

$\begin{array}{lr}\text { RG } & 512 \\ \text { OW } & 83.400 \text { usec }\end{array}$

IE $300.0 \mathrm{~K}$

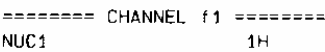

NUC1 $1 \mathrm{H}$

$\begin{array}{ll}\text { PL1 } & 10.10 \text { use } \\ \text { SFO1 } & 3.00 \mathrm{~dB}\end{array}$

Sh1. $400.1326008 \mathrm{MHz}$

F2 - Processing parameters

$\begin{array}{lc}\text { SI } & 16384 \\ \text { SF } & 400.1300091 \\ \text { WOW } & \text { MHZ }\end{array}$

$\begin{array}{cc}\text { WOW } & E M \\ 5 S B & 0 \\ B & 0.10\end{array}$

$\begin{array}{lc}\angle \mathrm{B} & 0.10 \mathrm{H} \\ \mathrm{GB} & 0 \\ { }^{\circ} \mathrm{C} & 1.00\end{array}$

10 NMR plot parameters

$\begin{array}{ll}\text { CX } & 20.00 \mathrm{~cm} \\ C Y & \end{array}$

$\begin{array}{lr}\text { CY } & 8.00 \mathrm{~cm} \\ \text { F1P } & 10.500 \mathrm{pPm} \\ \text { F1 } & 4201.37 \mathrm{~Hz}\end{array}$

$\begin{array}{ll}F 1 & 10.500 \mathrm{DPm} \\ F 2 \mathrm{P} & -0.500 \mathrm{Ppm} \\ F 2 & -200.07 \mathrm{~Hz}\end{array}$

PPMCM $\quad 0.55000 \mathrm{ppm} / \mathrm{cm}$ 
C13 spectrum of

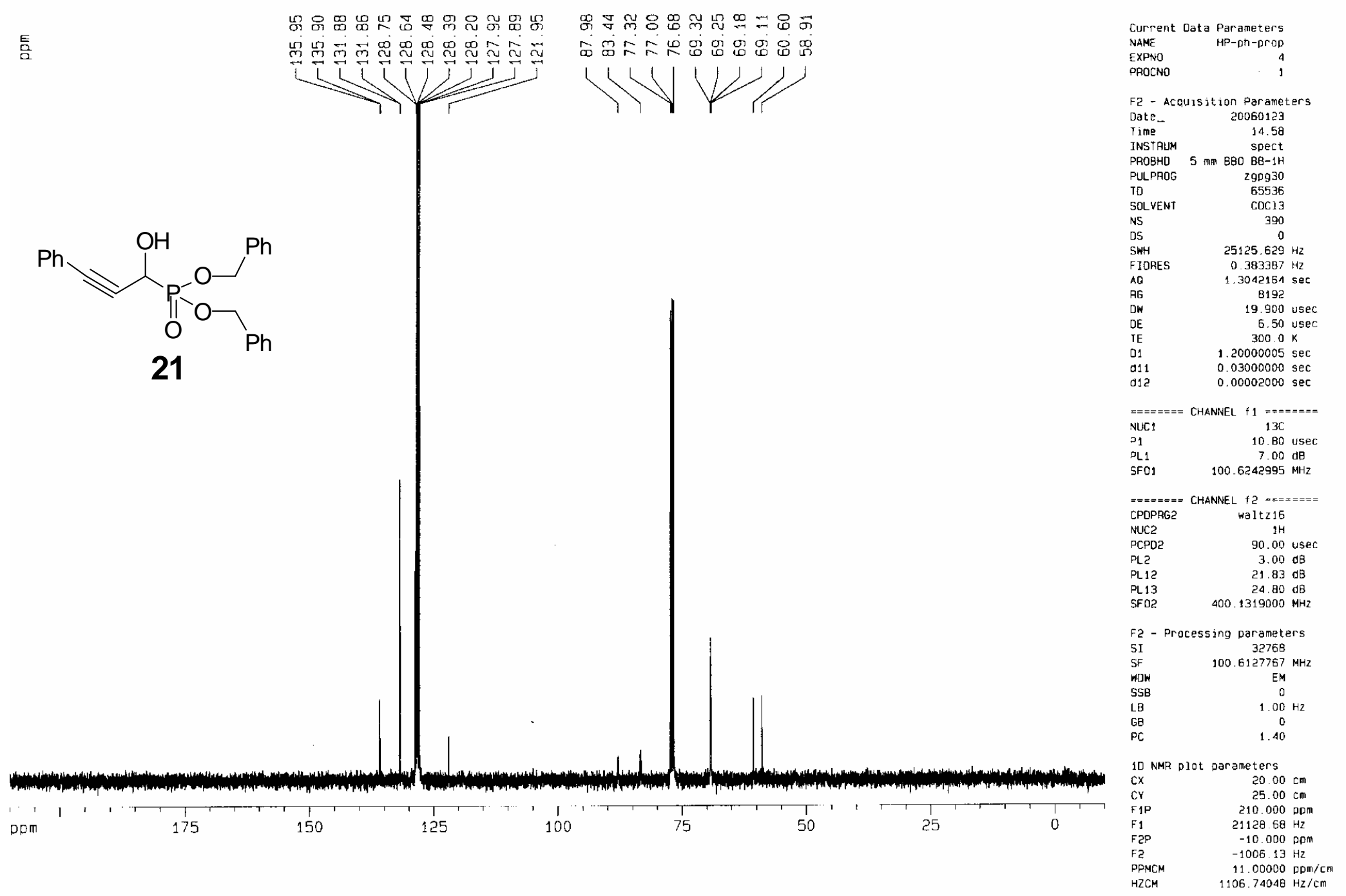

